\title{
Variational Inequalities with Lack of Ellipticity Part I: Optimal Interior Regularity and Non-degeneracy of the Free Boundary
}

\author{
Donatella Danielli, Nicola Garofalo, \\ \& SANDRO SALSA
}

\begin{abstract}
This paper is the first part of a program aimed at studying the regularity of sub-elliptic free boundaries. In the setting of Carnot groups we establish the optimal interior regularity of the solution to the obstacle problem in terms of the Folland-Stein non-isotropic class $\Gamma^{1,1}$. This result constitutes the sub-elliptic counterpart of the classical $C^{1,1}$ regularity for Laplace equation. We also prove non-degeneracy properties of the solution and of its free boundary.
\end{abstract}

\section{INTRODUCTION}

The study of the classical obstacle problem, initiated in the 60's with the pioneering works of G. Stampacchia, H. Lewy, J. L. Lions, has led over a period of three decades to beautiful and deep developments in calculus of variations and partial differential equations. The crowning achievement has been the development, due to L. Caffarelli, of the theory of free boundaries. For an assigned bounded domain $\Omega \subset \mathbb{R}^{n}$, one seeks to solve the variational inequality: given $f \in H^{-1}(\Omega)$, find $u \in \mathbf{K}_{\psi}$ such that

$$
a(v, v-u) \geq\langle f, v-u\rangle \quad \text { for all } v \in \mathbf{K}_{\psi} .
$$

Here,

$$
a(u, v)=\int_{\Omega}\langle A(x) \nabla u, \nabla v\rangle d x
$$

denotes the quadratic form on $H^{1}(\Omega)$ associated with an assigned uniformly elliptic matrix $A(x)=\left(a_{i j}(x)\right)$ with entries in $L^{\infty}(\Omega)$. Given an obstacle $\psi \in H^{1}(\Omega)$, 
satisfying $\psi \leq 0$ on $\partial \Omega$ (in the sense of $H^{1}(\Omega)$ ), one defines the convex set $\mathbf{K}_{\psi}=\left\{v \in H_{o}^{1}(\Omega) \mid v \geq \psi\right.$ on $\Omega$ in $\left.H^{1}(\Omega)\right\}$.

The above problem has a unique variational solution $u$, see [29]. Under additional regularity assumptions on $A(x)$ such solution possesses optimal interior smoothness properties. For instance, when $a_{i j}(x) \equiv \delta_{i j}$, then it is well-known that the optimal interior regularity of $u$ is expressed in terms of its membership to $H_{\text {loc }}^{2, \infty}(\Omega)$, or, equivalently, $u \in C_{\text {loc }}^{1,1}(\Omega)$.

In several problems ranging from geometry and optimal control [3], [41], to mechanical engineering or robotics [11], one is naturally led to the study of variational inequalities such as (1.1), but with a quadratic form (1.2) in which the matrix $A$ fails to be uniformly elliptic, or even elliptic at every point. A significant example is given by the matrix

$$
A=A(x, y, t)=\left(\begin{array}{ccc}
1 & 0 & 2 y \\
0 & 1 & -2 x \\
2 y & -2 x & 4\left(x^{2}+y^{2}\right)
\end{array}\right), \quad(x, y, t) \in \mathbb{R}^{3} .
$$

Since the lowest eigenvalue of $A(x, y, t)$ is $\lambda_{1} \equiv 0$ (the other two being $\lambda_{2} \equiv$ 1 , and $\left.\lambda_{3}=1+4\left(x^{2}+y^{2}\right)\right)$, this matrix fails to be elliptic at every point $(x, y, t) \in$ $\mathbb{R}^{3}$, although it is positive semi-definite. The reader can easily check that, given the vector fields

$$
X_{1}=\frac{\partial}{\partial x}+2 y \frac{\partial}{\partial t}, \quad X_{2}=\frac{\partial}{\partial y}-2 x \frac{\partial}{\partial t},
$$

the quadratic form (1.2) can presently be written as follows

$$
\begin{aligned}
a(u, v) & =\int_{\Omega}\langle A(x, y, t) \nabla u, \nabla v\rangle d x d y d t \\
& =\int_{\Omega}\langle X u, X v\rangle d x d y d t
\end{aligned}
$$

where we have denoted by $X u=\left(X_{1} u, X_{2} u\right)$ the "gradient" of the function $u$ with respect to the system $X=\left\{X_{1}, X_{2}\right\}$, and we have let $\langle X u, X v\rangle=X_{1} u X_{1} v+$ $X_{2} u X_{2} v$.

This example has three distinctive aspects. First, if we endow $\mathbb{R}^{3}$ with the non-abelian group law

$$
g \circ g^{\prime}=(x, y, t) \circ\left(x^{\prime}, y^{\prime}, t^{\prime}\right)=\left(x+x^{\prime}, y+y^{\prime}, t+t^{\prime}+2\left(x^{\prime} y-x y^{\prime}\right)\right),
$$

and we denote by $L_{g}\left(g^{\prime}\right)=g \circ g^{\prime}$ the corresponding left-translation, then $\left(\mathbb{R}^{3}, \circ\right)$ becomes a Lie group for which $X_{1}, X_{2}$, and $T=\partial / \partial t$, constitute a basis of the Lie algebra $\mathfrak{h}_{1}$ of all vector fields on $\mathbb{R}^{3}$ which are invariant with respect to $L_{g}$. 
Indicating, in fact, with $L_{g_{*}}$ the differential of $L_{g}$, it is easy to recognize that $L_{g_{*}}(\partial / \partial x)=X_{1}, L_{g_{*}}(\partial / \partial y)=X_{2}$, and that $L_{g_{*}}(\partial / \partial t)=T$. Long known to physicists as the Weyl group, and subsequently re-christened by mathematicians the Heisenberg group $\mathbb{U}^{1}$, such Lie group is the prototype, and in fact a model of paramount importance, of a large class of Lie groups that owe their name to the foundational paper of Carathéodory [9] on Carnot thermodynamics, and which play a distinguished role in analysis, geometry, mathematical physics, and in the applied sciences. Secondly, the Heisenberg algebra $\mathfrak{h}_{1}$ admits a stratification of step 2. By this we mean that it can be decomposed in the direct sum of two vector spaces $\mathfrak{h}_{1}=V_{1} \oplus V_{2}$, where $V_{1}=\mathbb{R}_{x, y}^{2}$, and $V_{2}=\mathbb{R}_{t}$. The important feature of such decomposition lies in the commutation relation $\left[X_{1}, X_{2}\right]=-4 T$, which shows that the system $X=\left\{X_{1}, X_{2}\right\}$ suffices to generate the whole algebra $\mathfrak{h}_{1}$. Thanks to $\left[X_{1}, X_{2}\right]=-4 T$, one has $\left[V_{1}, V_{1}\right]=V_{2}$, whereas $\left[V_{1}, V_{2}\right]=\{0\}$. The Heisenberg group is thus a stratified Lie group, which is nilpotent of step two, in short, a Carnot group of step two. The fact that one needs to resort to the commutator $\left[X_{1}, X_{2}\right]$ to recover the missing $T$ direction suggests that one should associate to (1.3) the anisotropic dilations $\delta_{\lambda}(x, y, t)=\left(\lambda x, \lambda y, \lambda^{2} t\right)$, rather than the standard Euclidean ones. That this guess is correct is easily seen by verifying that the two vector fields $X_{1}, X_{2}$ are homogeneous of degree one with respect to $\left\{\delta_{\lambda}\right\}_{\lambda>0}$. Thirdly, since the underlying manifold of $\mathbb{Q}^{1}$ is simply $\mathbb{R}^{3}$, it is easily recognized that Lebesgue measure is a left- (and right-) invariant Haar measure on the Lie group $\mathbb{\boxplus}^{1}$. It is clear that, if we denote $d H(g)=d x d y d t$, then $d\left(H \circ \delta_{\lambda}\right)(g)=\lambda^{4} d H(g)$, so that the correct dimension associated with $\mathbb{\boxplus}^{1}$ is $Q=4$. A natural norm is given by the so-called group gauge

$$
N(g)=\left(\left(x^{2}+y^{2}\right)^{2}+t^{2}\right)^{1 / 4},
$$

which is clearly homogeneous of degree one with respect to $\left\{\delta_{\lambda}\right\}_{\lambda>0}$. This allows to define a left-invariant distance on $\mathbb{a}^{1}$ by letting $d\left(g, g^{\prime}\right)=N\left(g^{-1} \circ g^{\prime}\right)$. The relative balls $B(g, R)=\left\{g^{\prime} \in \mathbb{Q}^{1} \mid d\left(g^{\prime}, g\right)<R\right\}$ have Haar measure given by $|B(g, R)|=\omega R^{4}$, where $\omega>0$ is a constant independent of $g \in \mathbb{a}^{1}$. Despite such translation invariance of their volume, the non-abelian group law (inherited by the product in $\mathbb{C}^{2}$ ) produces a strong distortion of the balls, and of the relative geometry. As a consequence, the basic properties of the latter are extremely different, and much harder to grasp, then those of Euclidean spaces.

The Euler-Lagrange equation of the variational integrand in the right-hand side of $(1.3)$ is given by

$$
\begin{aligned}
\mathcal{L} u & =\left(X_{1}^{2}+X_{2}^{2}\right) u \\
& =\frac{\partial^{2} u}{\partial x^{2}}+\frac{\partial^{2} u}{\partial y^{2}}+4\left(x^{2}+y^{2}\right) \frac{\partial^{2} u}{\partial t^{2}}+4 \frac{\partial}{\partial t}\left(y \frac{\partial u}{\partial x}-x \frac{\partial u}{\partial y}\right)=0 .
\end{aligned}
$$


Such equation costitutes the prototype of the general class studied in this paper. The second order differential operator $\mathcal{L}$ in (1.4) is known as the (real part of) the Kohn-Spencer sub-Laplacian on $\mathbb{t}^{1}$. Since the characteristic form associated with it is precisely the matrix $A(x, y, t)$ introduced above, $\mathcal{L}$ fails to be elliptic at every point of $\mathbb{R}^{3}$. However, the commutation relation $\left[X_{1}, X_{2}\right]=-4 T$ implies, in view of Hörmander's celebrated theorem [28], that it be hypoelliptic. Although we will not use in a direct way the sub-elliptic estimates in [28], the hypoellipticity of the relevant operator will play an important role in our study.

In partial differential equations Carnot groups first implicitly appeared in Kolmogorov's pioneering note [30], and many years later in H. Lewy's famous paper

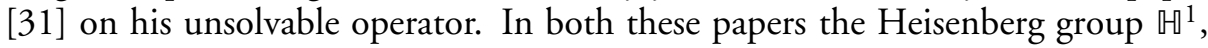
and the generators of its algebra $X_{1}$ and $X_{2}$ introduced in (1.3), are lurking in the background. In 1967 Hörmander proved his fundamental theorem [28] stating that, given a system $X_{o}, X_{1}, \ldots, X_{m}$ of smooth vector fields in $\mathbb{R}^{n}$ (or on a connected Riemannian manifold $M^{n}$ ), the finite rank condition

$$
\operatorname{rank} \operatorname{Lie}\left\{X_{o}, X_{1}, \ldots, X_{m}\right\} \equiv n
$$

is sufficient for the hypoellipticity of the partial differential operator $L=\sum_{j=1}^{m} X_{j}^{2}+$ $X_{o}$. This class includes trivially all elliptic and parabolic operators (with smooth coefficients), but in the important case when $m<n-1$, as the above example of the Heisenberg group shows, its elements fail to be either one.

The previous discussion should serve as an introduction, and hopefully a motivation, for the study undertaken in this paper. Having set up the stage, the question arises of what are the properties, both analytic and geometric, of the solution to the minimization problem which, similarly to the classical obstacle problem, is associated with the degenerate "energy" in (1.3). More specifically, does the solution $u$ have any degree of smoothness? If so, then what is its interior optimal regularity? What is the maximum growth of $u$ near the coincidence set $\{u=\varphi\}$ ? What is the size of the free boundary $\partial\{u=\varphi\}$ ? And, finally, what are the regularity properties, if any, of the latter? It should be clear to the reader that, in view of the global lack of ellipticity of (1.3), these questions cannot be answered within the classical framework and one needs to develop an appropriate theory which take into account the subtle geometric features of the problems at hand. The present paper constitutes only a first step in this program, our ultimate goal being the development of a theory of free boundaries for a class of variational inequalities which are modelled on the above example. The setting will be that of a general Carnot group. One of our main objectives here is to establish the analogue of the optimal $C_{\text {loc }}^{1,1}$ regularity from the elliptic theory for the solution to the relevant obstacle problem. Due to the degeneracy of the energy, however, one cannot hope for full smoothness of the solution since we are now forced to measure it in terms of the non-isotropic Lipschitz classes introduced by Folland and Stein [19], [18], [20]. We also prove some basic non-degeneracy properties 
of the solution, and of the free boundary. In a future study we plan to analyze the regularity properties of the latter.

To the reader familiar with the classical theory of variational inequalities and free boundaries it will be clear that such results constitute the counterpart of those established in the papers [32] $\left(C^{1, \alpha}\right.$ regularity with $\left.0<\alpha<1\right)$ and [23], [1] $\left(C^{1,1}\right.$ regularity). Our approach is strongly inspired to Caffarelli's revisitation [4] of the obstacle problem, see also [5]. There are, however, several new difficulties which cannot be dealt with by the classical ideas. For instance, in [4] the quadratic polynomials, the global solutions of $\Delta u=1$, play a crucial role in many respects. One important aspect is that if for such a $u$ one takes $v=D_{j} u$, then thanks to the commutativity of $D_{j}$ and $\Delta$, the function $v$ is harmonic. Another subtle fact is that the level sets of $u$ are spheres, and such are also the level sets of the fundamental solution of $\Delta$ vanishing at infinity. These features, and several others, simply fail in the degenerate setting of this paper. A major obstacle is represented by the fact that the involved vector fields $X_{1}, \ldots, X_{m}$ do not commute. As a consequence, for a solution of $\mathcal{L} u=1$ the function $v=X_{j} u$ is not harmonic (i.e., a solution of $\mathcal{L} u=0$ ), and one can no longer apply those Euclidean arguments based on commutation. Next, to circumvent the obstruction due to the lack of spherical symmetry we have to resort to a strange, yet remarkable symmetry result, which was established in [12], see Theorem 5.5 and the proof of Theorem 5.4. A third serious obstruction, which does not exist in the elliptic theory, is connected with the presence of characteristic points on the free boundary. Since we do not study the regularity of the latter in this paper, we will not discuss this question any further. We also mention another aspect which, in a sense, has a more basic character. It is well-known that in the classical theory of variational inequalities the fine differentiability properties of Lipschitz functions play a fundamental role. It is thus to be expected that an equally important role will have related properties of functions which are Lipschitz continuous with respect to the metric associated to a system of non-commuting vector fields. Unfortunately, this problem is hard and a complete solution in a general setting is still presently lacking, see the discussion in Section 2. In the process of proving Theorem 4.3 we needed a suitable quantitative version of the classical theorem of Rademacher-Stepanov for maps $f$ from a Carnot group to $\mathbb{R}$. There exists a well-known general result due to Pansu [35] (see Theorem 2.2 below) which is concerned with Lipschitz maps between two Carnot groups, but for reasons that we explain in Section 2 such result did not seem to suffice for our purposes. We have thus established two versions of the Rademacher-Stepanov theorem which, besides being instrumental to the present paper, also have an obvious independent interest, see Theorems 2.5 and 2.7, and also Remark 2.8. These results are connected to Pansu Theorem 2.2 below, (notice that in our case the target Carnot group is simply $(\mathbb{R},+)$ ). However, the full statement of our Theorem 2.7 is not contained in Pansu's result, and neither is Theorem 2.5 since Pansu does not deal with $L^{p}$ integrability. 
Having mentioned Hörmander's result, the experts might naturally wonder why we are focusing on the however geometrically important case of sub-Laplacians on a Carnot group, rather than attacking directly the study of the more general Hörmander type operators $\mathcal{L}=\sum_{j=1}^{m} X_{j}^{2}$. The motivation is twofold. On one hand, the structure of such groups plays a crucial role in the development of the theory of stratified Taylor polynomials, carried by Folland and Stein [20], which we heavily use to establish the interior regularity. A counterpart of such theory for the general Hörmander case presently constitutes, to the best of our knowledge, an interesting open question. Deeply connected to this, is another basic open problem: that of the best polynomial approximation of a harmonic function (a solution to the given sub-Laplacian). Again, thanks to the stratified polynomials we are able to nicely deal with this delicate point in the group setting, but there is at the moment no known analogue for Hörmander type operators. For both aspects, one will presumably have to look deeper into the Rothschild-Stein lifting theorem [36], but this endeavor will entail a non-trivial amount of work. Also, in view of the lifting theorem, it is natural to first develop a theory in the Carnot group setting. One should also take into account that the analysis of this setting is quite technically involved, and contains most of the difficulties of the general case.

We close this introduction with a description of the organization of the paper. Section 2 is devoted to establishing the above mentioned results of RademacherStepanov type, see Theorems 2.5 and 2.7. In Section 3 we prove that if the obstacle is in $\Gamma^{0, \alpha}, 0<\alpha \leq 1$, then so is the solution to the relative variational inequality, see Theorem 3.7. In Section 4 we study the optimal regularity of the solution. We show that when the obstacle belongs to the class $\Gamma^{1,1}$, then so does the solution, see Theorem 4.3. Finally, in Section 5 we prove further basic properties of a normalized solution of the obstacle problem. We should mention that perhaps it came as a surprise to us that such properties are valid in the degenerate setting of this paper. Theorem 5.3 provides an optimal bound for the degenerate gradient of the solution. In Theorem 5.4 we obtain an estimate which controls the maximum growth of the solution away from the free boundary. Finally, Theorem 5.6 states that the set where the solution is above the obstacle has uniform positive density with respect to the intrinsic metric at every free boundary point. As a corollary of the latter property, we show that the free boundary has measure zero, see Corollary 5.7, therefore at least in a measure theoretic sense it is well-behaved. In the Appendix at the end of the paper we have collected some basic known results which are used in the course of the proofs.

Acknowledgement. The present paper was completed while the first two authors were visiting the Institut Mittag-Leffler, during the special year on Potential Theory and Non-linear Partial Differential Equations. They gratefully acknowledge their indebtness to the organizers of the program and to the Institut for the invitation and for the gracious hospitality. We thank Pierre Pansu for useful comments concerning Section 2, and for bringing to our attention reference [45]. We also 
thank Roberto Monti and Francesco Serra Cassano for sending us a copy of their paper [34], and Serguei Vodop'yanov for providing us with a copy of [45].

\section{TheOrEMS OF RADEMACHER-STEPANOV TYPE}

To understand the fine properties of the solution $u$ to a variational inequality such as (1.1), but with lack of ellipticity, it is crucial to have a theorem of RademacherStepanov type for the horizontal derivatives $X_{1} u, \ldots, X_{m} u$ (see (2.2) for the appropriate definition). The objective of this section is to establish such result. We will in fact obtain a stronger $L^{p}$ version of the latter. The main results are Theorems 2.5 and 2.7 .

We begin introducing the relevant ambients and their many properties. A Carnot group $\mathbf{G}$ is a simply connected Lie group whose Lie algebra $\mathfrak{g}$ admits a nilpotent stratification of step $r$, i.e., there exist vector subspaces $V_{1}, \ldots, V_{r}$ of $\mathfrak{g}$, such that $\mathfrak{g}=V_{1} \oplus \cdots \oplus V_{r}$, with $\left[V_{1}, V_{j}\right]=V_{j+1}$ for $1 \leq j \leq r-1$, and $\left[V_{1}, V_{r}\right]=\{0\}$. By definition, the order of an element in $V_{j}$ is $j$. Every Carnot group is naturally equipped with a family of non-isotropic dilations defined by

$$
\delta_{\lambda}(g)=\exp \circ \Delta_{\lambda} \circ \exp ^{-1}(g), \quad g \in \mathbf{G},
$$

where exp $: \mathfrak{g} \rightarrow \mathbf{G}$ is the exponential map and $\Delta_{\lambda}: \mathfrak{g} \rightarrow \mathfrak{g}$ is defined by

$$
\Delta_{\lambda}\left(X_{1}+\cdots+X_{r}\right)=\lambda X_{1}+\cdots+\lambda^{r} X_{r}
$$

We assume that a scalar product $\langle\cdot, \cdot \cdot\rangle$ is given on $\mathfrak{g}$ for which the $V_{j}^{\prime} s$ are mutually orthogonal. If $m=\operatorname{dim}\left(V_{1}\right)$, we fix an orthonormal basis $X=\left\{X_{1}, \ldots, X_{m}\right\}$ of $V_{1}$, and continue to indicate with $X$ the corresponding system of left-invariant vector fields on $\mathbf{G}$. In a Carnot group the exponential map exp $: \mathfrak{g} \rightarrow \mathbf{G}$ is a global analytic diffemorphism. For a given function $f: \mathbf{G} \rightarrow \mathbb{R}$ the action of $X_{j}$ on $f$ is specified by the equation

$$
X_{j} f(g)=\lim _{t \rightarrow 0} \frac{f\left(g \exp \left(t X_{j}\right)\right)-f(g)}{t}=\left.\frac{d}{d t} f\left(g \exp \left(t X_{j}\right)\right)\right|_{t=0} .
$$

We now recall the important Baker-Campbell-Hausdorff formula, see, e.g., [42]

$$
\exp Z \exp Z^{\prime}=\exp \left(Z+Z^{\prime}+\frac{1}{2}\left[Z, Z^{\prime}\right]+\cdots\right), \quad Z, Z^{\prime} \in \mathfrak{g},
$$

where the dots indicate a finite linear combination of terms containing commutators of order two and higher. For $Z \in \mathfrak{g}$ consider the map $\theta_{Z}: \mathfrak{g} \rightarrow \mathfrak{g}$ given by

$$
\theta_{Z}\left(Z^{\prime}\right)=Z+Z^{\prime}+\frac{1}{2}\left[Z, Z^{\prime}\right]+\cdots
$$


where the right-hand side is given by the Baker-Campbell-Hausdorff sum in (2.3). If we endow the Lie algebra $\mathfrak{g}$ with the polynomial group law

$$
Z \circ Z^{\prime}=\theta_{Z}\left(Z^{\prime}\right),
$$

then we can identify the group $\mathbf{G}$ with $\mathfrak{g}$ via the exponential map.

The sub-Laplacian associated with $X$ is the second-order partial differential operator on $\mathbf{G}$ given by

$$
\mathcal{L}=\sum_{j=1}^{m} X_{j}^{2}
$$

By the assumption on the Lie algebra one immediately sees that the system $X$ satisfies the previously recalled finite rank condition, therefore thanks to Hörmander's theorem [28] the operator $\mathcal{L}$ is hypoelliptic. However, when $r>1$ it fails to be elliptic, and the loss of regularity is measured by the step $r$ of the stratification of $\mathfrak{g}$. For a function $u$ on $\mathbf{G}$ we let $X u=\left(X_{1} u, \ldots, X_{m} u\right)$, and set $|X u|=\left(\sum_{j=1}^{m}\left(X_{j} u\right)^{2}\right)^{1 / 2}$. We denote by $d H$ the push-forward of Lebesgue measure on $\mathfrak{g}$ via the exponential map. Such $d H$ defines a bi-invariant Haar measure on G. One has $d H\left(\delta_{\lambda}(g)\right)=\lambda^{Q} d H(g)$, so that the number $Q=\sum_{j=1}^{r} j \operatorname{dim} V_{j}$ plays the role of a dimension with respect to the group dilations. For this reason $Q$ is called the homogeneous dimension of $\mathbf{G}$. The topological dimension of $\mathrm{G}$ is instead the smaller number $N=\sum_{j=1}^{r} \operatorname{dim} V_{j}$. If we denote by $X_{j}^{*}$ the adjoint of $X_{j}$ in $L^{2}(\mathbf{G})$, then it is easy to see that $X_{j}^{*}=-X_{j}$. This shows that $\mathcal{L}=-\sum_{j=1}^{m} X_{j}^{*} X_{j}$, and therefore $\mathcal{L}$ is a divergence form operator on the Riemannian manifold $\mathbf{G}$ whose quadratic form is globally defined, non-ellitpic when $r>1$, symmetric and positive semi-definite.

We recall that a piecewise $C^{1}$ curve $\gamma:[0, T] \rightarrow \mathbf{G}$ is called horizontal if, whenever it exists, the tangent vector $\gamma^{\prime}(t)$ in $\gamma(t)$ satisfies the following constraint

$$
\left\langle\gamma^{\prime}(t), \xi\right\rangle^{2} \leq \sum_{j=1}^{m}\left\langle X_{j}(\gamma(t)), \xi\right\rangle^{2},
$$

for every cotangent vector $\xi$ in $\gamma(t)$. The horizontal length of $\gamma$ is by definition $\ell_{h}(\gamma)=T$. Thanks to the fundamental accessibility theorem of Chow [13], the stratification assumption on the Lie algebra $\mathfrak{g}$ implies that, given any two points $g, g^{\prime} \in \mathbf{G}$, there exists a horizontal curve $\gamma:[0, T] \rightarrow \mathbf{G}$, such that $\gamma(0)=g$, $\gamma(T)=g^{\prime}$. Thereby, if we denote by $\mathcal{H}\left(g, g^{\prime}\right)$ the collection of all horizontal curves joining $g$ to $g^{\prime}$, then we have $\mathcal{H}\left(g, g^{\prime}\right) \neq \emptyset$. The Carnot-Carathéodory distance between $g$ and $g^{\prime}$ is defined by

$$
\rho\left(g, g^{\prime}\right)=\inf \left\{\ell_{h}(\gamma) \mid \gamma \in \mathcal{H}\left(g, g^{\prime}\right)\right\} .
$$

One easily checks, see e.g. [24], the similarity relation

$$
\rho\left(\delta_{\lambda}(g), \delta_{\lambda}\left(g^{\prime}\right)\right)=\lambda \rho\left(g, g^{\prime}\right), \quad g, g^{\prime} \in \mathbf{G}, \lambda>0 .
$$


The Euclidean distance to the origin $|\cdot|$ on $\mathfrak{g}$ induces a homogeneous pseudonorm $|\cdot|_{\mathfrak{g}}$ on $\mathfrak{g}$ and (via the exponential map) one on the group $\mathbf{G}$ in the following way (see [18], [20]). For $\xi \in \mathfrak{g}$, with $\xi=\xi_{1}+\cdots+\xi_{r}, \xi_{i} \in V_{i}$, we let

$$
|\xi|_{\mathfrak{g}}=\left(\sum_{i=1}^{r}\left|\xi_{i}\right|^{2 r ! / i}\right)^{2 r !}
$$

and then define $|g|_{\mathbf{G}}=|\xi|_{\mathfrak{g}}$ if $\mathfrak{g}=\exp \xi$. The pseudo-norm defines a pseudodistance on $\mathbf{G}$

$$
d\left(\boldsymbol{g}, \boldsymbol{g}^{\prime}\right)=\left|\mathfrak{g}^{-1} \mathcal{g}^{\prime}\right|_{\mathbf{G}}
$$

The pseudo-distance (2.6) is equivalent to the Carnot-Carathéodory distance $\rho(\cdot, \cdot)$ generated by the system $X$, i.e., there exists a constant $C=C(\mathbf{G})>0$ such that

$$
C \rho\left(g, g^{\prime}\right) \leq d\left(g, g^{\prime}\right) \leq C^{-1} \rho\left(g, g^{\prime}\right), \quad g, g^{\prime} \in \mathbf{G},
$$

see [43]. If $B(g, R)=\left\{g^{\prime} \in \mathbf{G} \mid d\left(g, g^{\prime}\right)<R\right\}$, then by left-translation and dilation it is easy to see that the Haar measure of $B(g, R)$ is

$$
|B(g, R)|=\omega R^{Q},
$$

where $\omega=|B(e, 1)|$, with $e$ indicating the group identity. A similar translation invariance holds for the metric balls $B_{\rho}(g, R)$. The number $Q=\sum_{i=1}^{r} i \operatorname{dim} V_{i}$ is the homogeneous dimension of $\mathbf{G}$ related to the non-isotropic dilations, see [18].

As we mentioned in the introduction, the regularity results in this paper will be formulated in terms of some anisotropic Lipschitz classes, introduced by Folland and Stein [19], [20]. We next give the relevant definition.

Definition 2.1. Let $D \subset \mathbf{G}$ be a bounded open set and $0<\alpha \leq 1$. A bounded function $f: D \rightarrow \mathbb{R}$ is said to belong to the class $\Gamma^{0, \alpha}(D)$ if there exists a positive constant $L_{\alpha}>0$ such that

$$
\left|f(g)-f\left(g^{\prime}\right)\right| \leq L_{\alpha} d\left(g, g^{\prime}\right)^{\alpha}, \quad g, g^{\prime} \in D .
$$

Here, $d\left(g, g^{\prime}\right)$ represents the non-isotropic pseudo-distance on $\mathbf{G}$ generated by the natural gauge, see (2.6). The norm on the space $\Gamma^{0, \alpha}(D)$ is given by

$$
\|f\|_{\Gamma^{0, \alpha}(D)}=\|f\|_{L^{\infty}(D)}+\sup _{g, g^{\prime} \in D} \frac{\left|f(g)-f\left(g^{\prime}\right)\right|}{d\left(g, g^{\prime}\right)^{\alpha}} .
$$

A function $f \in \Gamma^{0,1}(D)$ is said to belong to the class $\Gamma^{1, \alpha}(D)$ if for every $j=$ $1, \ldots, m$ the derivative $X_{j} f$ exists in $D$ and moreover $X_{j} f \in \Gamma^{0, \alpha}(D)$. 
The meaning of the symbols $\Gamma_{\text {loc }}^{0, \alpha}(D), \Gamma_{\text {loc }}^{1, \alpha}(D)$ is the obvious one. Thus, e.g., $u \in \Gamma_{\text {loc }}^{0, \alpha}(D)$ if for every $\omega \subset \subset D$ one has $u \in \Gamma^{0, \alpha}(\omega)$. If $F \subset \mathbf{G}$ denotes a bounded closed set, by $f \in \Gamma^{1, \alpha}(F)$ we mean that $f$ coincides on the set $F$ with a function $g \in \Gamma^{1, \alpha}(D)$, where $D$ is a bounded open set containing $F$. The condition (2.9) in the Definition 2.1 measures the smoothness of $f$ in the intrinsic pseudo-metric $d\left(g, g^{\prime}\right)$ on $\mathbf{G}$ associated with the system $X=\left\{X_{1}, \ldots, X_{m}\right\}$ (of course, using the distance $\rho\left(g, g^{\prime}\right)$, instead of $d\left(g, g^{\prime}\right)$, in view of (2.7) would produce the same functional classes). The corresponding regularity of $f$ with respect to the underlying Riemannian metric $d_{R}\left(g, g^{\prime}\right)$ of $G$ is much lower (depending on the step $r$ of the group), since one has locally for some constant $C=C(\mathbf{G})>0$

$$
C d_{R}\left(g, g^{\prime}\right) \leq d\left(g, g^{\prime}\right) \leq C^{-1} d_{R}\left(g, g^{\prime}\right)^{1 / r} .
$$

After these preliminaries we turn to the main concern of this section. In his foundational paper [35] Pansu proved the following remarkable theorem for maps $f: \mathbf{G} \rightarrow \mathbf{G}^{\prime}$ between two Carnot groups.

Theorem 2.2 (Pansu's theorem of Rademacher-Stepanov type). Let $\mathbf{G}, \mathbf{G}^{\prime}$ be two Carnot groups, and consider open sets $\Omega \subset \mathbf{G}, \Omega^{\prime} \subset \mathbf{G}^{\prime}$. If $f: \Omega \rightarrow \Omega^{\prime}$ is a Lipschitz map with respect to the relative intrinsic metrics, i.e., there exists a constant $L>0$ such that

$$
d^{\prime}\left(f\left(g_{1}\right), f\left(g_{2}\right)\right) \leq L d\left(g_{1}, g_{2}\right), \quad g_{1}, g_{2} \in \Omega,
$$

then the differential of $f, D f(g)$, exists for $d H$-a.e. point $g \in \Omega$.

The notion of differential of a map between Carnot groups was introduced by Pansu himself. One says that $f$ is Pansu differentiable at $g \in \mathbf{G}$ if the maps

$$
f_{\lambda}=\delta_{\lambda^{-1}}^{\prime} \circ L_{f(g)^{-1}}^{\prime} \circ f \circ L_{g} \circ \delta_{\lambda}
$$

converge locally uniformly to a homomorphism $D f(g): \mathbf{G} \rightarrow \mathbf{G}^{\prime}$, as $\lambda \rightarrow 0$. Here, $L_{g}(h)=g h$ denotes the operator of left-translation on $\mathbf{G}$, whereas we have denoted by $L_{g^{\prime}}^{\prime}=g^{\prime} h^{\prime}$ the analogous operator on $\mathbf{G}^{\prime}$. Similarly, $\delta_{\lambda}$ and $\delta_{\lambda}^{\prime}$ respectively denote the dilations on $\mathbf{G}$ and $\mathbf{G}^{\prime}$.

For our purposes, besides the existence $d H$-a.e. of the differential, we also need to know that the latter belong to $L^{\infty}$, but Theorem 2.2 does not contain such quantitative information. Our first approach was to try to extract it directly from the original proof in [35]. However, on one hand it is not always easy for the analyst to translate geometric arguments into the more familiar language of estimates. On the other hand, an explicit discussion of the case in which the target group be $\mathbf{G}^{\prime}=(\mathbb{R},+)$ does not appear in [35]. These considerations prompted us to look for an "analyst" proof. In a classical 1941 note, L. Cesari [10] showed that for functions $f: \mathbb{R}^{2} \rightarrow \mathbb{R}$ the requirement that the (weak) derivatives belong 
to $L^{p}$ for some $p>2$ suffices to guarantee the existence of the differential of $f$ at a.e. point. Subsequently, A. P. Calderón [6] extended this result to any dimension, and established a sharp version of the latter in terms of Young functions and Orlicz spaces. This result, combined with a duality argument, gives a different proof, and in fact a stronger quantitative version, of the Rademacher-Stepanov theorem. For additional references the reader should see Theorem 1, ch. 8 of [38], or the more elementary approach in Theorem 1, ch. 6.2 of [16], and also the classical book [17].

In Theorem 2.5 we establish a generalization of the results in [10], [6], to maps from a Carnot group $G$ to $(\mathbb{R},+)$. We then combine Theorem 2.5 with the following Theorem 2.3, to obtain the full quantitative version of the RademacherStepanov theorem, see Theorem 2.7. We mention that Theorem 2.3 was obtained independently in [25] and [22].

Theorem 2.3. Let $\Omega \subset \mathbb{R}^{n}$ be an open set, bounded in the Carnot-Carathéodory metric $d(x, y)$ associated with a system $X=\left\{X_{1}, \ldots, X_{m}\right\}$ of Lipschitz vector fields in $\mathbb{R}^{n}$. If for $x, y \in \Omega$ one has for a constant $L>0$

$$
|f(x)-f(y)| \leq \operatorname{Ld}(x, y),
$$

then the weak derivatives of $f$ along the vector fields $X_{1}, \ldots, X_{m}$ belong to $L^{\infty}(\Omega)$.

We recall that, given $f \in L_{\text {loc }}^{1}\left(\mathbb{R}^{n}\right)$, we say that a function $F_{j} \in L_{\text {loc }}^{1}\left(\mathbb{R}^{n}\right)$ is the weak derivative of $f$ along the vector field $X_{j}$ if for every $\psi \in C_{o}^{\infty}\left(\mathbb{R}^{n}\right)$ one has

$$
\int_{\mathbb{R}^{n}} F_{j}(x) \psi(x) d x=\int_{\mathbb{R}^{n}} f(x) X_{j}^{*} \psi(x) d x .
$$

Here, if $X_{j}=\sum_{k=1}^{n} b_{k j}(x) \partial / \partial x_{k}$, we have denoted by $X_{j}^{*}$ the formal adjoint in $L^{2}\left(\mathbb{R}^{n}\right)$ of $X_{j}$, i.e., $X_{j}^{*}=-\sum_{k=1}^{n} \partial / \partial x_{k}\left(b_{k j}(x) \cdot\right)$. In a Carnot group one has $X_{j}^{*}=-X_{j}$, whenever $X=\left\{X_{1}, \ldots, X_{m}\right\}$ is a system of generators of the Lie algebra, thereby $F_{j}$ is the weak derivative of $f$ if

$$
\int_{\mathrm{G}} F_{j}(g) \psi(g), d H(g)=-\int_{\mathrm{G}} f(g) X_{j} \psi(g), d H(g),
$$

for any $\psi \in C_{o}^{\infty}(\mathbf{G})$. In the remaining part of this section, in order to distinguish the weak from the strong derivatives, we will adopt the notation $\bar{X}_{j} f$ for the weak derivative of $f$ with respect to $X_{j}$, and use $X_{j} f$ for the strong derivatives defined by (2.2). Given an open set $\Omega \subset \mathbf{G}$ we denote with $\mathcal{L}^{1, p}(\Omega), 1 \leq p \leq \infty$, the space of those distributions $f \in L^{p}(\Omega)$ such that $\bar{X} f \in L^{p}(\Omega)$. Such space is endowed with the natural norm

$$
\|f\|_{\mathcal{L}^{1, p}(\Omega)}=\|f\|_{L^{p}(\Omega)}+\|\bar{X} f\|_{L^{p}(\Omega)} .
$$


We now use the exponential map exp $: \mathfrak{g} \rightarrow \mathbf{G}$ to define analytic functions $\xi_{i}: \mathbf{G} \rightarrow V_{i}, i=1, \ldots, r$, through the equation

$$
g=\exp \left(\xi_{1}(g)+\xi_{2}(g)+\cdots+\xi_{r}(g)\right),
$$

where $\xi(g)=\xi_{1}(g)+\cdots+\xi_{r}(g)$ is such that $g=\exp (\xi(g))$. The coordinates of the first-component $\xi_{1}(g)$ in the basis $X_{1}, \ldots, X_{m}$ will be denoted by $x_{1}=$ $x_{1}(g), \ldots, x_{m}=x_{m}(g)$, i.e.,

$$
x_{j}(g)=\left\langle\xi_{1}(g), X_{j}\right\rangle \quad j=1, \ldots, m,
$$

and we set $x(g)=\left(x_{1}(g), \ldots, x_{m}(g)\right) \in \mathbb{R}^{m}$. We will need the following simple, yet crucial, proposition.

Proposition 2.4. Let $\mathbf{G}$ be a Carnot group. One has

$$
X_{i}\left(x_{j}\right)=\delta_{i j}, \quad \mathcal{L} x_{j}=0, \quad i, j=1, \ldots, m .
$$

Proof. Let $g=\exp (\xi(g))$, with $\xi(g)=\xi_{1}(g)+\cdots+\xi_{r}(g)$. From (2.3) we obtain for $t \in \mathbb{R}$

$$
x_{j}\left(g \exp t X_{i}\right)=x_{j}(g)+t \delta_{i j} .
$$

From the latter equation and from (2.2) the conclusion follows immediately.

In the next theorem the role of the Euclidean dimension in the results in [10] and [6] is played by the exponent $Q$ of volume growth of the intrinsic balls. We emphasize that it would be very interesting for the applications to have a version of Theorem 2.5 below for more general vector fields of Hörmander type, but for the latter one has to develop a substitute of the Folland-Stein stratified Taylor formula [20]. We plan to come back to this question in a future study.

Theorem 2.5 ( $L^{p}$-Rademacher-Stepanov type theorem). Let $\mathbf{G}$ be a Carnot group of step $r$ with homogeneous dimension $Q=\sum_{j=1}^{r} j \operatorname{dim} V_{j}$. If $f \in \mathcal{L}_{\operatorname{loc}}^{1, p}(\mathbf{G})$ for some $Q<p \leq \infty$, then the horizontal gradient $X f=\left(X_{1} f, \ldots, X_{m} f\right)$ exists $d H$ a.e. (and of course it coincides with the weak horizontal gradient of $f$ ). Furthermore, one has for $\mathrm{dH}-$ a.e. $g_{o} \in \mathbf{G}$

$$
\lim _{g \rightarrow g_{o}} \frac{f(g)-f\left(g_{o}\right)-\sum_{j=1}^{m}\left(x_{j}(g)-x_{j}\left(g_{o}\right)\right) X_{j} f\left(g_{o}\right)}{d\left(g, g_{o}\right)}=0 .
$$

Proof. We can assume without restriction that $Q<p<\infty$. By assumption, the weak derivatives of $f, \bar{X}_{j} f$, belong to $L_{\mathrm{loc}}^{p}(\mathbf{G})$, hence

$$
\bar{X} f(g)=\left(\bar{X}_{1} f(g), \ldots, \bar{X}_{m} f(g)\right)
$$


is finite at $d H$ - a.e. $g \in \mathbf{G}$. By this observation, and by the Lebesgue differentiation theorem in a space of homogeneous type (see [40]), we infer the existence of a $E \subset \mathbf{G}$ of $d H$-measure zero, such that for every $g_{o} \in \mathbf{G} \backslash E$

$$
\lim _{r \rightarrow 0} \frac{1}{\left|B\left(g_{o}, r\right)\right|} \int_{B\left(g_{o}, r\right)}\left|\bar{X}_{j} f\left(g^{\prime}\right)-\bar{X}_{j} f\left(g_{o}\right)\right|^{p} d H\left(g^{\prime}\right)=0, \quad j=1, \ldots, m .
$$

We next fix $g_{o} \in \mathbf{G} \backslash E$, and use the following basic representation theorem (see [33], or also [8] and [21] for general Hörmander vector fields)

$$
\left|F(g)-F_{B}\right| \leq C \int_{B\left(g_{o}, 3 r\right)} \frac{\left|\bar{X} F\left(g^{\prime}\right)\right|}{d\left(g, g^{\prime}\right)^{Q-1}} d H\left(g^{\prime}\right)
$$

valid for any function $F \in \mathcal{L}_{\text {loc }}^{1, p}(\mathbf{G})$, for $d H$-a.e. $g \in B=B\left(g_{o}, r\right)$. From the latter we obtain when $Q<p<\infty$, and therefore $1<p^{\prime}<Q /(Q-1)$,

$$
\begin{aligned}
\left|F(g)-F\left(g_{o}\right)\right| \leq C\left(\int_{B\left(g_{o}, 3 r\right)} \mid\right. & \left.\left.\bar{X} F\left(g^{\prime}\right)\right|^{p} d H\left(g^{\prime}\right)\right)^{1 / p} \\
& \times\left(\int_{B\left(g_{o}, 3 r\right)} \frac{1}{d\left(g, g^{\prime}\right)^{(Q-1) p^{\prime}}} d H\left(g^{\prime}\right)\right)^{1 / p^{\prime}} .
\end{aligned}
$$

A calculation gives

$$
\left(\int_{B\left(g_{o}, 3 r\right)} \frac{1}{d\left(g, g^{\prime}\right)^{(Q-1) p^{\prime}}} d H\left(g^{\prime}\right)\right)^{1 / p^{\prime}} \leq C \frac{r}{\left|B\left(g_{o}, 3 r\right)\right|^{1 / p}},
$$

hence

$$
\left|F(g)-F\left(g_{o}\right)\right| \leq C r\left(\frac{1}{\left|B\left(g_{o}, 3 r\right)\right|} \int_{B\left(g_{o}, 3 r\right)}\left|\bar{X} F\left(g^{\prime}\right)\right|^{p} d H\left(g^{\prime}\right)\right)^{1 / p} .
$$

At this point we take $F(g)=f(g)-f\left(g_{o}\right)-\sum_{j=1}^{m}\left(x_{j}(g)-x_{j}\left(g_{o}\right)\right) \bar{X}_{j} f\left(g_{o}\right)$. Clearly, $F\left(g_{o}\right)=0$, whereas from the proof of Proposition 2.4 we have $\bar{X}_{i} F(g)=$ $\bar{X}_{i} f(g)-\bar{X}_{i} f\left(g_{o}\right)$. Inserting such $F$ in (2.14) we find

$$
\begin{aligned}
\mid f(g) & -f\left(g_{o}\right)-\sum_{j=1}^{m}\left(x_{j}(g)-x_{j}\left(g_{o}\right)\right) \bar{X}_{j} f\left(g_{o}\right) \mid \\
\leq & C r\left(\frac{1}{\left|B\left(g_{o}, 3 r\right)\right|} \int_{B\left(g_{o}, 3 r\right)}\left|\bar{X} f\left(g^{\prime}\right)-\bar{X} f\left(g_{o}\right)\right|^{p} d H\left(g^{\prime}\right)\right)^{1 / p}
\end{aligned}
$$


In (2.15) we now choose $g=g_{t}=g_{o} \exp \left(t X_{i}\right)$, where $i \in\{1, \ldots, m\}$ is fixed. Since $x_{j}\left(g_{t}\right)=x_{j}\left(g_{o}\right)+t \delta_{i j}$, we conclude that $f\left(g_{t}\right)-f\left(g_{o}\right)-\sum_{j=1}^{m}\left(x_{j}\left(g_{t}\right)-x_{j}\left(g_{o}\right)\right) \bar{X}_{j} f\left(g_{o}\right)=f\left(g_{t}\right)-f\left(g_{o}\right)-t \bar{X}_{i} f\left(g_{o}\right)$.

Noting that $d\left(g_{t}, g_{o}\right) \leq t$, one obtains from (2.14)

$$
\begin{aligned}
& \left|f\left(g_{t}\right)-f\left(g_{o}\right)-t \bar{X}_{i} f\left(g_{o}\right)\right| \\
& \quad \leq C t\left(\frac{1}{\left|B\left(g_{o}, 3 t\right)\right|} \int_{B\left(g_{o}, 3 t\right)}\left|\bar{X} f\left(g^{\prime}\right)-\bar{X} f\left(g_{o}\right)\right|^{p} d H\left(g^{\prime}\right)\right)^{1 / p} .
\end{aligned}
$$

Dividing by $t$, letting $t \rightarrow 0$ in the latter inequality, and keeping in mind (2.2), we conclude that $X_{i} f\left(g_{o}\right)$ exists and equals $\bar{X}_{i} f\left(g_{o}\right)$. Repeating this argument for each $i=1, \ldots, m$, we infer the first part of the theorem. Once this has been established we return to $(2.15)$, in which we can now substitute $\bar{X}_{j} f\left(g_{o}\right)$ with $X_{j} f\left(g_{o}\right)$. Choosing $r=d\left(g, g_{o}\right)$ in (2.15), we obtain

$$
\begin{gathered}
\frac{\left|f(g)-f\left(g_{o}\right)-\sum_{j=1}^{m}\left(x_{j}(g)-x_{j}\left(g_{o}\right)\right) X_{j} f\left(g_{o}\right)\right|}{d\left(g, g_{o}\right)} \\
\leq C\left(\frac{1}{\left|B\left(g_{o}, 3 d\left(g, g_{o}\right)\right)\right|} \int_{B\left(g_{o}, 3 d\left(g, g_{o}\right)\right)}\left|X f\left(g^{\prime}\right)-X f\left(g_{o}\right)\right|^{p} d H\left(g^{\prime}\right)\right)^{1 / p} .
\end{gathered}
$$

Since the limit of the right-hand side as $d\left(g, g_{o}\right) \rightarrow 0$ is zero, we see that (2.13) holds. This completes the proof.

We note explicitly that when $G^{\prime}=(\mathbb{R},+)$, then the definition (2.10) of Pansu differential of a function $f: \mathbf{G} \rightarrow \mathbb{R}$ becomes

$$
D f(g)(h)=\lim _{\lambda \rightarrow 0^{+}} \frac{f\left(g \delta_{\lambda}(h)\right)-f(g)}{\lambda},
$$

the limit being locally uniform in $h \in \mathrm{G}$. In the next proposition we give a characterization of formula (2.17) in the case in which $f$ is sufficiently smooth.

Proposition 2.6. Let $\mathbf{G}$ be a Carnot group of step $r$, and $f \in C_{o}^{1}(\mathbf{G})$. The Pansu differential of $f$ is given by the formula

$$
D f(g)(h)=\sum_{j=1}^{m} x_{j}(h) X_{j} f(g)=\langle x(h), X f(g)\rangle, \quad g, h \in \mathbf{G} .
$$


Proof. We prove Proposition 2.6 in the case in which G is a Carnot group of step 2, and then indicate the changes which are necessary to cover the case of arbitrary step. If $\mathbf{G}$ is of step 2 , then $\mathfrak{g}=V_{1} \oplus V_{2}$. Denote with $Y_{1}, \ldots, Y_{k}$ an orthonormal basis of the second layer $V_{2}$. We will indicate with $y(g)=$ $\left(y_{1}(g), \ldots, y_{k}(g)\right)$ the projection onto $V_{2}$ of the exponential coordinates of $g$, i.e.,

$$
y_{\ell}(g)=\left\langle\xi_{2}(g), Y_{\ell}\right\rangle \quad \ell=1, \ldots, k .
$$

The exponential coordinates of a point $g \in \mathbf{G}$ are thus given by

$$
\left(x_{1}(g), \ldots, x_{m}(g), y_{1}(g), \ldots, y_{k}(g)\right) \text {. }
$$

For $i, j=1, \ldots, m, \ell=1, \ldots, k$, let $b_{i j}^{\ell}$ denote the group constants, i.e.,

$$
\left[X_{i}, X_{j}\right]=\sum_{\ell=1}^{k} b_{i j}^{\ell} Y_{\ell}
$$

If $f: \mathbf{G} \rightarrow \mathbb{R}$, then the following useful formula for the derivative $X_{j} f(g)$ in exponential coordinates holds

$$
X_{j} f(g)=\frac{\partial f}{\partial x_{j}}(g)+\frac{1}{2} \sum_{\ell=1}^{k}\left(\sum_{i=1}^{m} b_{i j}^{\ell} x_{i}(g)\right) \frac{\partial f}{\partial y_{\ell}}(g) .
$$

To prove (2.20) we recall the definition (2.2) of $X_{j} f(g)$. Let $g=\exp \xi(g)$, with $\xi(g)=\xi_{1}(g)+\xi_{2}(g)$. Using (2.3) one obtains

$$
g \exp \left(t X_{j}\right)=\exp \left(\xi_{1}(g)+t X_{j}+\xi_{2}(g)+\frac{t}{2}\left[\xi_{1}(g), X_{j}\right]\right) .
$$

From (2.19) we find

$$
\left[\xi_{1}(g), X_{j}\right]=\sum_{\ell=1}^{k}\left(\sum_{i=1}^{m} b_{i j}^{\ell} x_{i}(g)\right) Y_{\ell},
$$

and therefore

$$
\begin{aligned}
f\left(g \exp \left(t X_{j}\right)\right)=f\left(x_{1}(g), \ldots,\right. & x_{j}(g)+t, \ldots, x_{m}(g), y_{1}(g) \\
& \left.+\frac{t}{2} \sum_{i=1}^{m} b_{i j}^{1} x_{i}(g), \ldots, y_{k}(g)+\frac{t}{2} \sum_{i=1}^{m} b_{i j}^{k} x_{i}(g)\right) .
\end{aligned}
$$


Differentiating the latter equation with respect to $t$, and setting $t=0$, we obtain (2.20).

We next compute the Pansu differential of $f$. From the definition (2.1), using (2.3) we obtain

$$
\begin{gathered}
=f\left(\exp \left(\xi_{1}(g)+\lambda \xi_{1}(h)+\xi_{2}(g)+\lambda^{2} \xi_{2}(h)+\frac{\lambda}{2}\left[\xi_{1}(g), \xi_{1}(h)\right]\right)\right) \\
=f\left(x_{1}(g)+\lambda x_{1}(h), \ldots, x_{m}(g)+\lambda x_{m}(h), y_{1}(g)\right. \\
+\lambda^{2} y_{1}(h)+\frac{\lambda}{2} \sum_{i, j=1}^{m} b_{i j}^{1} x_{i}(g) x_{j}(h), \ldots, y_{k}(g) \\
\left.+\lambda^{2} y_{k}(h)+\frac{\lambda}{2} \sum_{i, j=1}^{m} b_{i j}^{k} x_{i}(g) x_{j}(h)\right) .
\end{gathered}
$$

Differentiating (2.21) with respect to $\lambda$, and setting $\lambda=0$, gives in view of (2.17)

$$
\begin{aligned}
D f(g)(h) & =\sum_{j=1}^{m}\left[\frac{\partial f}{\partial x_{j}}(g)+\frac{1}{2} \sum_{\ell=1}^{k}\left(\sum_{i=1}^{m} b_{i j}^{\ell} x_{i}(g)\right) \frac{\partial f}{\partial y_{\ell}}(g)\right] x_{j}(h) \\
& =\sum_{j=1}^{m} x_{j}(h) X_{j} f(g),
\end{aligned}
$$

where in the last equality we have used (2.20). This proves the proposition for a Carnot group of step 2.

In the case of arbitrary step one has to replace formula (2.20) with a sligthly more complicated one. Let $m_{s}=\operatorname{dim} V_{s}, s=1, \ldots, r$, and continue to indicate with $X_{1}, \ldots, X_{m}$ an orthonormal basis of $V_{1}$. We denote by $\left(x_{1}(g), \ldots, x_{r}(g)\right)$ the exponential coordinates of $g=\exp \left(\xi_{1}(g)+\cdots+\xi_{r}(g)\right)$, where for each $s=1, \ldots, r$ we have presently let $x_{s}(g)=\left(x_{s, 1}(g), \ldots, x_{s, m_{s}}(g)\right)$ for the componenets of $\xi_{1}(g)$ with respect to a fixed orthonormal basis of $V_{s}$. The following formula holds

$$
X_{j} f(g)=\frac{\partial f}{\partial x_{1, j}}+\sum_{s=2}^{r} \sum_{\ell=1}^{m_{s}} b_{s, \ell}^{j}\left(x_{1}, \ldots, x_{s-1}\right) \frac{\partial f}{\partial x_{s, \ell}}, \quad j=1, \ldots, m_{1},
$$

where each $b_{s, \ell}^{j}$ is a homogeneous polynomial of weighted degree $s-1$. By weighted degree we mean that, as previously mentioned, the layer $V_{s}, s=1, \ldots, r$, 
in the stratification of $\mathfrak{g}$ is assigned the formal degree $s$. Correspondingly, each homogeneous monomial $x_{1}^{\alpha_{1}} x_{2}^{\alpha_{2}} \cdots x_{r}^{\alpha_{r}}$, with multi-indices $\alpha_{s}=\left(\alpha_{1, s}, \ldots, \alpha_{m_{s}, s}\right)$, $s=1, \ldots, r$, is said to have weighted degree $p$ if $\sum_{s=1}^{r} s\left(\sum_{\ell=1}^{m_{s}} \alpha_{l, s}\right)=p$. Formula (2.22) is proved using (2.3), analogously to (2.20), and we leave the details to the interested reader. The remainder of the proof of the proposition proceeds now as for the case $r=2$.

As a consequence of Theorems 2.5 and 2.3 we obtain the following result which will play a crucial role in the subsequent part of this paper.

Theorem 2.7 (Horizontal Rademacher-Stepanov Theorem). Let $\Omega$ be an open subset of a Carnot group $\mathbf{G}$, and consider $f: \Omega \rightarrow \mathbb{R}$, with $f \in \Gamma^{0,1}(\Omega)$. There exists a set $E \subset \Omega$ of $d H$-measure zero such that the Pansu differential $D f(g)$ and the horizontal gradient $X f(g)=\left(X_{1} f(g), \ldots, X_{m} f(g)\right)$ exist for every $g \in \Omega \backslash E$, and

$$
D f(g)(h)=\sum_{j=1}^{m} x_{j}(h) X_{j} f(g)=\langle x(h), X f(g)\rangle, \quad \text { for every } h \in \mathbf{G} .
$$

Furthermore, $X f \in L^{\infty}(\Omega)$.

Proof. In view of Theorem 2.3, the assumption $f \in \Gamma^{0,1}(\Omega)$ implies that $f \in \mathcal{L}^{1, \infty}(\Omega)$. In particular, the weak horizontal gradient $\bar{X} f=\left(\bar{X}_{1} f, \ldots, \bar{X}_{m} f\right)$ belongs to $L^{\infty}(\mathbf{G})$, hence, in particular, it exists $d H$ - a.e. Thanks to Theorem 2.5 there exists $E \subset \Omega$ of $d H$-measure zero such that the ordinary horizontal gradient $X f(g)=\left(X_{1} f(g), \ldots, X_{m} f(g)\right)$ exists for every $g \in \Omega \backslash E$. A standard argument shows that $X f=\bar{X} f d H$-a.e. To complete the proof we are left with showing that (2.23) holds. Fix an open set $\omega \Subset \Omega$ and let $\psi \in C_{o}^{1}(\omega)$. The translation invariance of $d H$ implies for $\lambda>0$ small enough

$$
\begin{aligned}
\int_{\Omega} & \frac{f\left(g \delta_{\lambda}(h)\right)-f(g)}{\lambda} \psi(g) d H(g) \\
& =\int_{\Omega} f(g) \frac{\psi\left(g\left(\delta_{\lambda}(h)\right)^{-1}\right)-\psi(g)}{\lambda} d H(g) \\
& =\int_{\Omega} f(g) \frac{\psi\left(g\left(\delta_{\lambda}\left(h^{-1}\right)\right)\right)-\psi(g)}{\lambda} d H(g),
\end{aligned}
$$

where in the second equality we have used the fact $\left(\delta_{\lambda}(h)\right)^{-1}=\delta_{\lambda}\left(h^{-1}\right)$, which follows easily form (2.3). Thanks to (2.3), one has $x\left(h^{-1}\right)=-x(h)$. Inserting 
this information in (2.24), and passing to the limit we obtain

$$
\begin{aligned}
\lim _{\lambda \rightarrow 0^{+}} & \int_{\Omega} \frac{f\left(g \delta_{\lambda}(h)\right)-f(g)}{\lambda} \psi(g) d H(g) \\
& =\int_{\Omega} f(g) D \psi(g)\left(h^{-1}\right) d H(g) \\
& =-\int_{\Omega} f(g)\langle x(h), X \psi(g)\rangle d H(g),
\end{aligned}
$$

where in the last equality we have used Proposition 2.6. Recalling now (2.11), and integrating by parts in the right-hand side of (2.25), we conclude

$$
\begin{aligned}
\lim _{\lambda \rightarrow 0^{+}} & \int_{\Omega} \frac{f\left(g \delta_{\lambda}(h)\right)-f(g)}{\lambda} \psi(g) d H(g) \\
& =\int_{\Omega}\langle x(h), \bar{X} f(g)\rangle \psi(g) d H(g) \\
& =\int_{\Omega}\langle x(h), X f(g)\rangle \psi(g) d H(g) .
\end{aligned}
$$

Suppose now that $\left|f(g)-f\left(g^{\prime}\right)\right| \leq L d\left(g, g^{\prime}\right)$, for every $g, g^{\prime} \in \Omega$, then

$$
\left|f\left(g \delta_{\lambda}(h)\right)-f(g)\right| \leq L d\left(g \delta_{\lambda}(h), g\right)=L d\left(\delta_{\lambda}(h), e\right)=L \lambda d(h, e),
$$

where we have denoted with $e$ the identity in $\mathrm{G}$. This shows that the difference quotient in the integral in the left-hand side of the latter equality is bounded uniformly in $\lambda$. From Lebesgue dominated convergence theorem we finally infer

$$
\begin{aligned}
\int_{\Omega}\langle x & (h), X f(g)\rangle \psi(g) d H(g) \\
& =\lim _{\lambda \rightarrow 0^{+}} \int_{\Omega} \frac{f\left(g \delta_{\lambda}(h)\right)-f(g)}{\lambda} \psi(g) d H(g) \\
& =\int_{\Omega} \lim _{\lambda \rightarrow 0^{+}} \frac{f\left(g \delta_{\lambda}(h)\right)-f(g)}{\lambda} \psi(g) d H(g) \\
& =\int_{\Omega} D f(g) \psi(g) d H(g) .
\end{aligned}
$$

The latter equality proves (2.23) for $d H$-a.e. $g \in \omega$. The arbitrariness of $\omega$ implies the conclusion.

Remark 2.8. We mention that, after this paper was submitted, we received an interesting preprint from R. Monti and F. Serra Cassano [34] in which the authors, with completely different objectives than those in this paper, have obtained results similar to Theorems 2.5 and 2.7. Subsequently, it was brought to our attention by P. Pansu that analogous results were also obtained by S. Vodop'yanov in his interesting paper [45]. 
Next, we state an important converse to Theorem 2.7 which was established in [25]. This result will be used in Sections 3 and 4. The general setting will be the same of Theorem 2.3.

Theorem 2.9 ( $L^{\infty}$ Poincare inequality). Assume that the Carnot-Carathéodory balls are open in the topology of $\mathbb{R}^{n}$. Given a bounded open set $U \subset \mathbb{R}^{n}$ there exist $R_{o}=R_{o}(U)>0$, and $C=C(U)>0$, such that if $f \in \mathcal{L}^{1, \infty}\left(B\left(x_{o}, 3 R\right)\right)$, with $x_{o} \in U$ and $0<R<R_{o}$, then $f$ can be modified on a set of $d x$-measure zero in $\bar{B}=\bar{B}\left(x_{o}, R\right)$, so as to satisfy for every $x, y \in \bar{B}\left(x_{o}, R\right)$

$$
|f(x)-f(y)| \leq C d(x, y)\|f\|_{\mathcal{L}^{1, \infty}\left(B\left(x_{o}, 3 R\right)\right)} .
$$

If, furthermore, $f \in C^{\infty}\left(B\left(x_{o}, 3 R\right)\right)$, then in the right hand side of the previous inequality one can replace the term $\|f\|_{\mathcal{L}^{1, \infty}\left(B\left(x_{0}, 3 R\right)\right)}$ with $\|X f\|_{L^{\infty}\left(B\left(x_{0}, 3 R\right)\right)}$.

We note explicitly that the theorem asserts that every function $f \in$ $\mathcal{L}^{1, \infty}\left(B\left(g_{o}, 3 R\right)\right)$ has a representative which is Lipschitz continuous in $B\left(g_{o}, R\right)$ with respect to the metric $d$, i.e., continuing to denote with $f$ such representative, one has $f \in \Gamma^{0,1}\left(B\left(g_{o}, R\right)\right)$. We also observe that the limitation on the radius $R_{o}(U)$ in the statement of the theorem is merely dictated by a similar limitation on the validity of the doubling condition $\left|B\left(x_{o}, 2 r\right)\right| \leq C_{1}\left|B\left(x_{o}, r\right)\right|$. When the ambient space is a Carnot group G, then thanks to (2.8) the doubling condition holds globally in a trivial way, and one can thus take $U=\mathbf{G}$ and $R_{o}=\infty$. Theorems 2.3 and 2.9 together, completely characterize, in the general setting of Carnot-Carathéodory metrics for Lipschitz vector fields, the degenerate Sobolev space $\mathcal{L}^{1, \infty}(\Omega)$ as the space of metric Lipschitz functions.

\section{Regularity of the SOlution IN THE FOLLAND-STEIN LIPSCHITZ ClASSES}

The purpose of this section is to prove Theorem 3.7. The latter states that the solution to a general class of variational inequalities with non-elliptic integrands modelled on (1.3) belongs to the Folland-Stein Lipschitz class $\Gamma_{\mathrm{loc}}^{0, \alpha}$, with $0<\alpha \leq$ 1 , if so does the obstacle. Before doing this we need to state the relevant problem. For those known results which will be needed in the sequel the reader is referred to the Appendix in Section 6.

We fix a bounded domain $D \subset \mathbf{G}$ and a continuous function $\varphi: \bar{D} \rightarrow \mathbb{R}$, an obstacle, satisfying

$$
\max _{D} \varphi>0, \quad \varphi \leq 0 \quad \text { on } \partial D .
$$

Consider the closed convex set

$$
\mathbf{K}_{\varphi}=\left\{v \in \stackrel{o}{\mathcal{L}}^{1,2}(D) \mid v \geq \varphi \quad \text { a.e. in } D\right\},
$$

where $\stackrel{\mathcal{L}}{\mathcal{L}}^{1,2}(D)={\overline{C_{O}^{\infty}(D)}}^{\|\cdot\|_{\mathcal{L}^{1,2}(D)}}$. Our purpose is to study the variational inequality. 
Problem. Find $u \in \mathbf{K}_{\varphi}$ such that

$$
\int_{D}\langle X v, X(v-u)\rangle d H \geq 0
$$

for $v \in \mathbf{K}_{\varphi}$.

Since the quadratic form in (3.1) is coercive in the $m$-dimensional vector $\zeta=X u$, following the classical ideas in [32], [37], [29], one proves the existence of a unique solution to this problem in the degenerate Sobolev space $\mathcal{L}^{1,2}(D)$.

A function $w \in \mathcal{L}_{\text {loc }}^{1,2}(D)$ will be called $\mathcal{L}$-superharmonic in $D$ if for any $\zeta \in$ $C_{o}^{\infty}(D), \zeta \geq 0$, one has

$$
\int_{D}\langle X w, X \zeta\rangle d H \geq 0
$$

It is easy to see that the solution $u$ to the obstacle problem is $\mathcal{L}$-superharmonic in $D$. We have in fact the following.

Lemma 3.1. Let $u$ be the solution to the sub-elliptic obstacle problem in $D$, then $u$ is $\mathcal{L}$-superharmonic in $D$.

Proof. Consider the solution $u$ to problem (3.1). For any $\zeta \in C_{o}^{\infty}(D), \zeta \geq 0$ the function $v=u+\zeta$ belongs to the closed convex set $\mathbf{K}_{\varphi}$, thereby

$$
\int_{D}\langle X u, X \zeta\rangle d H \geq 0 .
$$

This shows that $u$ is $\mathcal{L}$-superharmonic in $D$.

Next, we consider the set in which $u$ is above the obstacle

$$
\Omega=\Omega(u, \varphi)=\{g \in D \mid u(g)>\varphi(g)\},
$$

and the coincidence set

$$
\Lambda=\Lambda(u, \varphi)=\{g \in D \mid u(g)=\varphi(g)\} .
$$

We would like to know that the former is open, whereas the latter is closed relatively to $D$. This information does follow in fact from the following basic result, which can be derived from the weak Harnack inequality in the Appendix, see Theorem 6.3. Since the arguments are similar to those in the classical elliptic case (see for instance the excellent monograph [26]), we omit the proof. The reader should also consult the paper by Hervé and Hervé [27].

Theorem 3.2. Let $u \in \mathcal{L}^{1,2}(D)$ be a $\mathcal{L}$-superharmonic function in $D$, then $u$ is locally essentially bounded from below and there exists a lower semi-continuous representative of $u$, which we continue to denote with the same letter, such that

$$
u\left(g_{o}\right)=\text { ess } \liminf _{g \rightarrow g_{o}} u(g)
$$


for every $g_{o} \in D$.

Since the obstacle $\varphi$ in (3.1) is locally bounded we also have the following property.

Theorem 3.3. Let $u$ be the solution to the obstacle problem (3.1), then $u$ is locally bounded in $D$.

We say that $u$ is $\mathcal{L}$-harmonic in $D$ if both $u$ and $-u$ are $\mathcal{L}$-superharmonic. The following result shows that the solution to the obstacle problem is $\mathcal{L}$-harmonic in the set $\Omega(u, \varphi)$ where $u$ is above the obstacle.

Lemma 3.4. For the solution to (3.1) one has the inclusion

$$
\operatorname{supp} \mathcal{L} \mathrm{u} \subset \Lambda(\mathrm{u}, \varphi) .
$$

In particular, we have $\mathcal{L} u=0$ in $\Omega(u, \varphi)$, and therefore by the hypoellipticity of $\mathcal{L}$ we conclude that $u \in C^{\infty}(\Omega(u, \varphi))$.

Proof. By Theorem 3.2 we know that the set $\Omega(u, \varphi)$ is open, therefore given $g_{o} \in \Omega(u, \varphi)$ there exists $B\left(g_{o}, r\right) \subset \Omega(u, \varphi)$. Fix $\eta \in C_{o}^{\infty}\left(B\left(g_{o}, r / 2\right)\right)$ and let $\varepsilon>0$ be such that

$$
v \stackrel{\text { def }}{=} u+\varepsilon \eta \geq \varphi .
$$

With this choice we have $v \in \mathbf{K}_{\varphi}$, therefore

$$
0 \leq \int_{D}\langle X u, X(v-u)\rangle d H=\varepsilon \int_{D}\langle X u, X \eta\rangle d H .
$$

The arbitrariness of $\eta$ shows that

$$
\int_{D}\langle X u, X \eta\rangle d H=0
$$

hence $\mathcal{L} u=0$ in $B\left(g_{o}, r / 2\right)$. This proves the desired inclusion.

Finally, we have the following generalization of a classical result due to Evans [15] and Vasilesco [44]. Again, its proof is based on Theorem 6.3, and thus we omit it referring the reader to that of Theorem 3.67 in [26]. in $D$.

Theorem 3.5. The solution $u$ to the sub-elliptic obstacle problem is continuous

In a sense to be made precise subsequently, on the set $\Lambda$ the function $u$ inherits the same smoothness properties of the obstacle $\varphi$. Our concern is the regularity of $u$ across the support of its sub-Laplacian. The answer is contained in the two Theorems 3.7 and 4.3. The remainder of this section is devoted to proving Theorem 3.7. We begin with a key lemma. 
Lemma 3.6. Suppose that for some $0<\alpha \leq 1$ one has $\varphi \in \Gamma^{0, \alpha}(\bar{D})$. Let $g_{o} \in$ $\Lambda$ and denote by $\omega(r)$ the modulus of continuity of $\varphi$ in a pseudo-ball $B\left(g_{o}, r\right) \subset$ $\bar{B}\left(g_{o}, r\right) \subset D$. There exist a constant $C>0$, depending on $\mathbf{G}$, such that

$$
\sup _{B\left(g_{o}, r\right)}(u-\varphi) \leq C \omega(r) .
$$

Proof. By definition one has for every $g \in B\left(g_{o}, r\right)$

$$
\left|\varphi(g)-\varphi\left(g_{o}\right)\right| \leq \omega(r) .
$$

Consider the function

$$
w(g)=u(g)-\varphi\left(g_{o}\right)+\omega(r),
$$

Thanks to Lemma 3.1, $w$ is $\mathcal{L}$-superharmonic in $D$. Moreover, (3.2) implies $w \geq 0$ in $B\left(g_{o}, r\right)$. We write $w=w_{1}+w_{2}$, where $w_{1}$ is the generalized solution to the Dirichlet problem

$$
\begin{cases}\mathcal{L} w_{1}=0 & \text { in } B\left(g_{o}, r\right) \\ w_{1}=w & \text { on } \partial B\left(g_{o}, r\right) .\end{cases}
$$

From Theorem 3.5 we know $u \in C(D)$, therefore also $w \in C(D)$. By Bony's maximum principle we have $0 \leq w_{1} \leq w$ in $B\left(g_{o}, r\right)$. Moreover, $w \geq w_{2}=$ $w-w_{1} \geq 0$. This implies

$$
w_{1}\left(g_{o}\right) \leq w\left(g_{o}\right)=u\left(g_{o}\right)-\varphi\left(g_{o}\right)+\omega(r)=\omega(r),
$$

since, by the hypothesis, $g_{o} \in \Lambda$. This inequality and Theorem 6.4 give for some $C=C(\mathbf{G})>0$

$$
\sup _{B\left(g_{o}, r / 2\right)} w_{1} \leq C \omega(r)
$$

Since

$$
\begin{cases}\mathcal{L} w_{2} \leq 0 & \text { in } B\left(g_{o}, r\right) \\ w_{2}=0 & \text { on } \partial B\left(g_{o}, r\right),\end{cases}
$$

by Theorem 6.2 we infer

$$
\sup _{B\left(g_{o}, r\right)} w_{2}=\sup _{\operatorname{supp} \mathcal{L} w_{2}} w_{2}=\sup _{\operatorname{supp} \mathcal{L} u} w_{2}
$$


which implies that

$$
\sup _{B\left(g_{o}, r\right)} w_{2}=w_{2}\left(g_{1}\right)
$$

for some $g_{1} \in \operatorname{supp} \mathcal{L} u$. By Lemma 3.4 we know supp $\mathcal{L} u \subset \Lambda$, so that in $g_{1}$ one has $u\left(g_{1}\right)=\varphi\left(g_{1}\right)$. This gives for $g \in B\left(g_{o}, r\right)$

$$
w_{2}(g) \leq w\left(g_{1}\right)=u\left(g_{1}\right)-\varphi\left(g_{1}\right)+\omega(r) \leq \omega(r) .
$$

From (3.4), (3.6) and (3.7) we conclude

$$
\sup _{B\left(g_{o}, r / 2\right)} w \leq C \omega(r)
$$

To finish the proof we observe that $w(g)=u(g)-\varphi(g)+\varphi(g)-\varphi\left(g_{o}\right)+$ $\omega(r)$, so that

$$
u(g)-\varphi(g)=w(g)-\left[\varphi(g)-\varphi\left(g_{o}\right)\right]-\omega(r) .
$$

From this observation and from (3.8) the conclusion readily follows.

Theorem 3.7. Suppose that the obstacle $\varphi$ belong to $\Gamma^{0, \alpha}(\bar{D})$ for some $0<\alpha \leq 1$, then the solution $u$ to the sub-elliptic obstacle problem can be modified on a set of $\mathrm{dH}$ measure zero in $D$ in such a way that the resulting function (which we still denote by $u)$ is in $\Gamma_{\mathrm{loc}}^{0, \alpha}(D)$.

Proof. We fix $h_{o} \in \partial \Omega$ and $\delta>0$ in such a way that $\bar{B}\left(h_{o}, \delta\right) \subset D$. Our aim is to prove the existence of a constant $C>0$ such that

$$
\left|u(g)-u\left(g^{\prime}\right)\right| \leq C d\left(g, g^{\prime}\right)^{\alpha}, \quad g, g^{\prime} \in B\left(h_{o}, \delta\right) .
$$

Let $g, g^{\prime} \in B\left(h_{o}, \delta\right)$. If both points belong to the coincidence set $\Lambda$, then there is nothing to prove. We assume next that $g, g^{\prime} \in \Omega$ and let $g_{o}, g_{o}^{\prime} \in \partial \Omega=$ $\partial \Lambda \subset \Lambda$ be such that

$$
d\left(g, g_{o}\right)=d(g, \partial \Omega)=\rho, \quad d\left(g^{\prime}, g_{o}^{\prime}\right)=d\left(g^{\prime}, \partial \Omega\right)=\rho^{\prime} .
$$

We distinguish two cases.

Case 1: $d\left(g, g^{\prime}\right)<\frac{1}{6} \max \left(\rho, \rho^{\prime}\right)$. Without loss of generality we assume $\max \left(\rho, \rho^{\prime}\right)=\rho$ and consider the function $w=u-\varphi\left(g_{o}\right)+C \omega(\rho)$, where $\omega(\rho)$ represents the modulus of continuity of $\varphi$ in $B\left(g_{o}, \rho\right)$. We notice that

$$
B\left(g, 3 d\left(g, g^{\prime}\right)\right) \subset B\left(g, \frac{\rho}{2}\right) \subset \Omega
$$


and therefore $w$ is $\mathcal{L}$-harmonic in $B(g,(\rho / 2))$. Applying Theorems 2.9 and 6.5 we find

$$
\begin{aligned}
\left|u(g)-u\left(g^{\prime}\right)\right| & =\left|w(g)-w\left(g^{\prime}\right)\right| \\
& \leq d\left(g, g^{\prime}\right)\|X w\|_{L^{\infty}\left(B\left(g, 3 d\left(g, g^{\prime}\right)\right)\right)} \\
& \leq d\left(g, g^{\prime}\right) \frac{C}{\rho} \sup _{B(g, 3 / 4 \rho)}|w| \\
& \leq d\left(g, g^{\prime}\right) \frac{C}{\rho} \sup _{B\left(g_{o}, 2 \rho\right)} w \leq C \frac{d\left(g, g^{\prime}\right)}{\rho^{1-\alpha}} \leq C d\left(g, g^{\prime}\right)^{\alpha},
\end{aligned}
$$

where in the second to the last inequality we have used (3.8).

Case $2: d\left(g, g^{\prime}\right) \geq \frac{1}{6} \max \left(\rho, \rho^{\prime}\right)$. Again we suppose without restriction that $\max \left(\rho, \rho^{\prime}\right)=\rho$. One has

$$
\left|u(g)-u\left(g^{\prime}\right)\right| \leq\left|u(g)-u\left(g_{o}\right)\right|+\left|u\left(g_{o}\right)-u\left(g_{o}^{\prime}\right)\right|+\left|u\left(g_{o}^{\prime}\right)-u\left(g^{\prime}\right)\right| .
$$

The term $\left|u\left(g_{o}\right)-u\left(g_{o}^{\prime}\right)\right|=\left|\varphi\left(g_{o}\right)-\varphi\left(g_{o}^{\prime}\right)\right|$ is estimated trivially by $d\left(g_{o}, g_{o}^{\prime}\right)^{\alpha}$. Since

$$
d\left(g_{o}, g_{o}^{\prime}\right) \leq d\left(g_{o}, g\right)+d\left(g, g^{\prime}\right)+d\left(g^{\prime}, g_{o}^{\prime}\right) \leq 13 d\left(g, g^{\prime}\right),
$$

we conclude

$$
\left|u\left(g_{o}\right)-u\left(g_{o}^{\prime}\right)\right| C d\left(g, g^{\prime}\right)^{\alpha} .
$$

To estimate $\left|u(g)-u\left(g_{o}\right)\right|$ we consider the function $h=u-u\left(g_{o}\right)$, which is $\mathcal{L}$-harmonic in $\Omega$. Applying Theorems 2.9 and 6.5 to the function $h$ in the ball $B(g, \rho)$ we obtain

$$
\begin{aligned}
\left|u(g)-u\left(g_{o}\right)\right| & \leq d\left(g, g_{o}\right) \sup _{B(g, \rho / 2)}|X h| \\
& \leq d\left(g, g_{o}\right) \frac{C}{\rho} \sup _{B(g, 5 / 4 \rho)} \| h \mid \\
& \leq d\left(g, g_{o}\right) \frac{C}{\rho} \sup _{B\left(g_{o}, 2 \rho\right)}|h| \\
& \leq d\left(g, g_{o}\right) \frac{C}{\rho}\left[\sup _{B\left(g_{o}, 2 \rho\right)}|u-\varphi|+\sup _{B\left(g_{o}, 2 \rho\right)}\left|\varphi-\varphi\left(g_{o}\right)\right|\right] \\
& \leq C \rho^{\alpha} \leq C d\left(g, g^{\prime}\right)^{\alpha} .
\end{aligned}
$$

Similarly, one obtains

$$
\left|u(g)-u\left(g_{o}\right)\right| \leq C d\left(g, g^{\prime}\right)^{\alpha} .
$$


Combining the above estimates we conclude

$$
\left|u(g)-u\left(g^{\prime}\right)\right| C d\left(g, g^{\prime}\right)^{\alpha} .
$$

Finally, the case in which $g \in \Omega$ and $g^{\prime} \in \Lambda$ is treated analogously to the previous ones. This completes the proof of (3.9) and thereby of the theorem.

The next theorem will play a crucial role in the remaining part of this paper.

Theorem 3.8. Assume that $\varphi \in \Gamma^{0,1}(\bar{D})$, then the solution of the sub-elliptic obstacle problem $u$ belongs to $\mathcal{L}_{\mathrm{loc}}^{1, \infty}(D)$. Furthermore, there exists a set $E \subset D$ of $d H$ measure zero such that the horizontal gradient $X u=\left(X_{1} u, \ldots, X_{m} u\right)$ exists in every $g \in D \backslash E$ and it coincides with the weak horizontal gradient.

Proof. The fact that $u \in \mathcal{L}_{\text {loc }}^{1, \infty}(D)$ follows from Theorem 2.3. The second part of the theorem follows from Theorem 2.7.

The following remark is an important consequence of Theorem 3.8

Remark 3.9. Suppose that the obstacle $\varphi \in \Gamma^{0,1}(\bar{D})$. By Theorem 3.8 we know that the continuous function $f=u-\varphi$ admits horizontal gradient $X f$ in $d H$-a.e. $g \in D$. Since on the other hand $f \geq 0$ in $D$ and $f(g)=0$ at every $g \in \Lambda$, we infer that must be $X u(g)=X \varphi(g)$ at $d H$-a.e. $g \in \Lambda$.

\section{INTERIOR Optimal REgULARITY OF THE SOLUTION}

With the results from Sections 2 and 3 in our hands, we now return to the study of the higher regularity of the solution of the obstacle problem. The following consequence of Theorem 1.42 in [20] will play a pervasive role in the sequel. As we mentioned in the introduction, the generalization of this result to a general system of Hörmander type presently constitutes a basic open question.

Theorem 4.1. Let $f \in \Gamma_{\text {loc }}^{1,1}(\mathbf{G})$. There exist constants $C, b>0$, depending only on $\mathbf{G}$, such that for every $g \in B\left(g_{o}, r\right)$

$$
\left|f(g)-T_{f}\left(g ; g_{o}\right)\right| \leq C r \sup _{g^{\prime} \in B\left(g_{o}, b r\right)}\left|X f\left(g^{\prime}\right)-X f\left(g_{o}\right)\right| .
$$

Here, we have denoted by

$$
T_{f}\left(g ; g_{o}\right)=f\left(g_{o}\right)+\sum_{j=1}^{m}\left(x_{j}(g)-x_{j}\left(g_{o}\right)\right) X_{j} f\left(g_{o}\right)
$$

the stratified Taylor polynomial of $f$, of homogeneous degree one, centered at $g_{o}$. 
Lemma 4.2. We assume that the obstacle $\varphi$ belongs to $\Gamma^{1,1}(\bar{D})$. Let $g_{o} \in \Lambda$ and denote by $\omega(r)$ the modulus of continuity of $X \varphi$ in a pseudo-ball $B\left(g_{o}, r\right)$. There exist constants $C, b>0$, depending on $\mathbf{G}$, such that if $\bar{B}\left(g_{o}, b r\right) \subset D$

$$
\sup _{B\left(g_{o}, r\right)}(u-\varphi) \leq \operatorname{Cr} \omega(b r) .
$$

Proof. We introduce the function

$$
T(g)=T_{\varphi}\left(g ; g_{o}\right)=\varphi\left(g_{o}\right)+\sum_{j=1}^{m}\left(x_{j}(g)-x_{j}\left(g_{o}\right)\right) X_{j} \varphi\left(g_{o}\right) .
$$

Thanks to Proposition 2.4, the following two basic properties hold

$$
\mathcal{L} T=0, \quad X T \equiv X \varphi\left(g_{o}\right), \quad \text { in } \mathbf{G} .
$$

Furthermore, Theorem 4.1 guarantees for every $r>0$ such that $B\left(g_{o}, r\right) \subset$ $\bar{B}\left(g_{o}, b r\right) \subset D$

$$
|\varphi(g)-T(g)| \leq \operatorname{Cr} \omega(b r) .
$$

From (4.3) we obtain for every $g \in B\left(g_{o}, r\right)$

$$
u(g) \geq \varphi(g) \geq T(g)-\operatorname{Cr} \omega(b r) .
$$

Define

$$
w(g)=u(g)-T(g)+C r \omega(b r) .
$$

From (4.4), (4.2), and from the $\mathcal{L}$-superharmonicity of $u$, one infers

$$
w \geq 0, \quad \mathcal{L} w \leq 0 \text { in } B\left(g_{o}, r\right) .
$$

Following the proof of Lemma 3.6 we write $w=w_{1}+w_{2}$, where $w_{1}$ is the generalized solution to the Dirichlet problem

$$
\begin{cases}\mathcal{L} w_{1}=0 & \text { in } B\left(g_{o}, r\right) \\ w_{1}=w & \text { on } \partial B\left(g_{o}, r\right)\end{cases}
$$

One presently obtains

$$
\sup _{B\left(g_{o}, r / 2\right)} w_{1} \leq \operatorname{Cr} \omega(b r)
$$


whereas, similarly to (3.6), we have

$$
\sup _{B\left(g_{o}, r\right)} w_{2}=w_{2}\left(g_{1}\right)
$$

for some $g_{1}$ such that $u\left(g_{1}\right)=\varphi\left(g_{1}\right)$. Recalling that $w_{2} \leq w$, we find

$$
\begin{aligned}
w_{2}\left(g_{1}\right) \leq w\left(g_{1}\right) & =u\left(g_{1}\right)-T\left(g_{1}\right)+C r \omega(b r) \\
& =\varphi\left(g_{1}\right)-T\left(g_{1}\right)+\operatorname{Cr} \omega(b r) \\
& \leq \operatorname{Cr} \omega(b r) .
\end{aligned}
$$

From (4.5), (4.6) and (4.7) we conclude

$$
\sup _{B\left(g_{o}, r / 2\right)} w \leq \operatorname{Cr} \omega(b r) .
$$

To finish the proof we observe that $w(g)=u(g)-\varphi(g)+\varphi(g)-T(g)+$ $\operatorname{Cr} \omega(b r)$, so that

$$
u(g)-\varphi(g)=w(g)-[\varphi(g)-T(g)]-\operatorname{Cr} \omega(b r) .
$$

From this observation, and from (4.3), (4.8), the conclusion follows.

Theorem 4.3. Suppose that $\varphi \in \Gamma^{1,1}(\bar{D})$, then the solution $u$ to the obstacle problem can be modified on a set of $d H$-measure zero in $D$ so that the resulting function is in $\Gamma_{\mathrm{loc}}^{1,1}(D)$. From this property, and from Theorem 2.3, we conclude that for every $\omega \subset \subset \subset$ one has $u \in \mathcal{L}^{2, \infty}(\omega)=\left\{f \in L^{\infty}(\omega) \mid X_{i} X_{j} f \in L^{\infty}(\omega)\right.$, $i, j=1, \ldots, m\}$.

Proof. The proof is similar to that of Theorem 3.7 so we only give few details. One thing that we need to observe preliminarily is that by assumption the horizontal gradient of the obstacle $X \varphi$ exists everywhere in $\bar{D}$ and defines a function in $\Gamma^{0,1}(\bar{D})$. Now, according to Remark 3.9 there exists a set $E \subset D$ of $d H$ measure zero such that $X u(g)=X \varphi(g)$ for every $g \in \Lambda \backslash E$. On the intersection of the exceptional set $E$ with the coincidence set $\Lambda$ we define $X u$ to be equal to $X \varphi$. In this way we have defined $X u$ everywhere on $\Lambda$, and hence on $D$. We cannot stress enough the importance of this step, which is made possible by Theorem 2.7. Our aim is to now prove that such extended function is in $\Gamma_{\text {loc }}^{0,1}(D)$. We proceed exactly as in the proof of Theorem 3.7 and distinguish the two cases considered there.

In case 1 we consider the function

$$
w=u-T+C_{1} \rho \omega(4 b \rho),
$$

where $T=T(g)$ is as in (4.1), $C_{1}$ and $b$ are the absolute constants in Theorem 4.1 , and $\omega(\rho)$ represents the modulus of continuity of $X \varphi$ on $B\left(g_{o}, \rho\right)$. By (4.1) 
and (4.2) we know that

$$
\mathcal{L} w=0 \quad \text { in } \quad B(g, \rho), \quad X w(g)=X u(g)-X \varphi\left(g_{o}\right),
$$

so that, applying Theorems 2.9 and 6.5, we find

$$
\begin{aligned}
& \left|X u(g)-X u\left(g^{\prime}\right)\right|=\left|X w(g)-X w\left(g^{\prime}\right)\right| \\
C & \leq d\left(g, g^{\prime}\right) \sup _{i, j=1, \ldots, m}\left\|X_{i} X j\right\|_{L^{\infty}\left(B\left(g, 3 d\left(g, g^{\prime}\right)\right)\right)} \\
& \leq \frac{C}{\rho^{2}} d\left(g, g^{\prime}\right) \sup _{B\left(g_{o}, 2 \rho\right)}|w| \\
& \leq \frac{C}{\rho} d\left(g, g^{\prime}\right) \rho \leq C d\left(g, g^{\prime}\right),
\end{aligned}
$$

where in the second to the last inequality we have used Lemma 4.2.

In case 2 we again proceed as in the proof of Theorem 3.7 except that we now consider the function $h=u-T$. The details are left to the reader.

Remark 4.4. Theorem 4.3 provides the optimal regularity of the solution $u$ to the sub-elliptic obstacle problem. In fact, across the free boundary $\partial \Omega$ the horizontal derivatives of order two $X_{i} X_{j} u$ cannot be continuous. To see this consider in the pseudo-ball of radius one centered at the group identity $B=B(e, 1) \subset \mathbf{G}$ the solution $\varphi$ to the Dirichlet problem $\mathcal{L} \varphi=-1, \varphi=0$ on $\partial B$. Let $u$ be the solution to the obstacle problem with obstacle $\varphi$. We know from Theorem 3.5 that $\mathcal{L} u=0$ in $\Omega=\Omega(u, \varphi)$, whereas on $B \backslash \bar{\Omega}$ one has $\mathcal{L} u=\mathcal{L} \varphi=-1$. This proves that at least one of the second order derivatives $X_{j} X_{j} u$ must be discontinuous.

The Heisenberg group. It is interesting to see the implications of Theorem 4.3 in the basic example of the Heisenberg group $\mathbb{Q}^{1}$ discussed in the introduction. It is well-known that the $t$-direction is the bad one, since one has a priori no control of the derivative $\partial / \partial t$. However, if we consider the solution $u$ to the obstacle problem associated with the quadratic form (1.3), then Theorem 4.3 states, in particular, that the mixed derivatives $X_{1} X_{2} u$ and $X_{2} X_{1} u$ belong to $L_{\text {loc }}^{\infty}$ across the free boundary. Thanks to the commutation relation $\left[X_{1}, X_{2}\right]=-4 \partial / \partial t$, this implies that $\partial u / \partial t \in L_{\mathrm{loc}}^{\infty}$. This highly non-trivial information will play an important role in the further understanding of the regularity of the free boundary.

\section{Non-Degeneracy Properties of the Solution and of THE FREE BOUNDARY}

After having established Theorems 3.7 and 4.3, we continue the study of the fine properties of the solution $u$ to the sub-elliptic obstacle problem (3.1), and prove some basic non-degeneracy of the latter and of its free boundary. The main results are Theorems 5.3, 5.4 and 5.6. These results, along with Theorem 4.3, constitute 
essential ingredients for the development of a theory of free boundaries. We start with the solution $u$ to (3.1), and normalize it by letting

$$
w=u-\varphi .
$$

Since $\mathcal{L} u \leq 0$ in $D$, then by Bony's strong maximum principle Theorem 6.2, $u$ cannot touch $\varphi$ at a point where $\mathcal{L} \varphi \geq 0$, so near the free boundary $\partial \Omega$ we should expect $\mathcal{L} \varphi<0$. This shows that in $\Omega$ one has

$$
\mathcal{L} w=\mathcal{L}(u-\varphi)=-\mathcal{L} \varphi>0 .
$$

Following [4], we normalize the problem by assuming $\mathcal{L} \varphi \equiv-1$. The advantage is that now the study of the regularity properties of the solution $w$, and of the free boundary $\partial \Omega$, is reduced to a local problem by looking at the so-called normalized solution. By this we mean a function $w$ which in the unit gauge pseudo-ball $B=B(e, 1) \subset \mathbf{G}$ satisfies the following properties:

(i) $w \geq 0$ in $B$.

(ii) $\mathcal{L} w=1$ on the set $\Omega(w)=\{g \in B \mid w(g)>0\}$.

We propose to study some properties of $w$ and the free boundary $\partial \Omega(w)$. An important remark is that if $w$ is a normalized solution of the sub-elliptic obstacle problem in the unit ball $B$, then the function

$$
w_{\lambda}=\frac{1}{\lambda^{2}} w \circ \delta_{\lambda}
$$

is a normalized solution in $B_{\lambda}=B(e, \lambda)$.

Proposition 5.1. There exists a constant $C>0$, depending on $\mathbf{G}$, such that the function $w$ and its $\Gamma^{1,1}$ norm in $B_{1 / 2}$ are bounded by $C$.

Proof. Consider the solution $\psi$ to the problem $\mathcal{L} \psi=1$ in $B, \psi=0$ on $\partial B$. By the hypoellipticity of $\mathcal{L}$, see [28], we have $\psi \in C^{\infty}(B)$. Let $u$ be the solution to the obstacle problem with obstacle $\psi$, then $u=w-\psi$. The conclusion follows by applying Theorem 4.3 to the function $u$.

Before proceeding with the study of the properties of the function $w$ we pause to establish some preliminary estimates which will be a first substitute for the explicit global solutions (quadratic polynomials) from the classical theory.

Lemma 5.2. Let $g_{o} \in \mathbf{G}$ and in the pseudo-ball $B\left(g_{o}, R\right)$ consider the Green function $G\left(g, g^{\prime}\right)$ for $\mathcal{L}$, and the solution

$$
\psi(\mathfrak{g})=\int_{B\left(g_{o}, R\right)} G\left(\mathfrak{g}, \mathfrak{g}^{\prime}\right) d H\left(g^{\prime}\right)
$$


to the problem $\mathcal{L} \psi=-1$ in $B\left(g_{o}, R\right), \psi=0$ on $\partial B\left(g_{o}, R\right)$. By Theorem 6.2 one has $\psi>0$ in $B\left(g_{o}, R\right)$. Moreover, there exists a constant $C>0$, depending only on G, such that for every $g \in B\left(g_{o}, R\right)$

$$
\psi(g) \leq C R^{2},
$$

and also

$$
\left|X \psi\left(g_{o}\right)\right| \leq C R .
$$

Proof. By left translation it is enough to establish the lemma in the case $g_{o}=$ $e$, the group identity. By Theorem 6.2 one has for every $g, g^{\prime} \in B\left(g_{o}, R\right)$

$$
G\left(g, g^{\prime}\right) \leq \Gamma\left(g, g^{\prime}\right),
$$

where $\Gamma\left(\mathfrak{g}, \mathfrak{g}^{\prime}\right)=\Gamma\left(\mathfrak{g}^{\prime}, \mathfrak{g}\right)$ denotes the global fundamental solution of $-\mathcal{L}$ in G constructed by Folland in [18]. We recall that if let $\Gamma^{*}(g)=\Gamma(g, e)$, then $\Gamma\left(g, g^{\prime}\right)=\Gamma^{*}\left(g^{-1} g^{\prime}\right)$. Furthermore, it was proved in [18] that, if $Q$ denotes the homogeneous dimension of $\mathbf{G}$, then the distribution $\Gamma^{*}$ is homogeneous of degree $2-Q$ with respect to the one-parameter group of non-isotropic dilations $\left\{\delta_{\lambda}\right\}_{\lambda>0}$. Therefore, the following estimates hold

$$
\frac{\alpha}{d\left(g, g^{\prime}\right)^{Q-2}} \leq \Gamma\left(g, g^{\prime}\right) \leq \frac{\beta}{d\left(g, g^{\prime}\right)^{Q-2}}, \quad g, g^{\prime} \in \mathbf{G}, g \neq g^{\prime},
$$

where we have let

$$
\alpha=\min _{g \in \partial B(e, 1)} \Gamma^{*}(g), \quad \beta=\max _{g \in \partial B(e, 1)} \Gamma^{*}(g) .
$$

Theorem 6.2 implies that $\alpha>0$. Using (5.1) we obtain

$$
\begin{aligned}
\psi(\mathfrak{g}) & \leq \int_{B(\mathfrak{g}, 2 R)} \Gamma\left(\mathfrak{g}, \mathfrak{g}^{\prime}\right) d H\left(\mathfrak{g}^{\prime}\right) \\
& \leq \sum_{k=0}^{\infty} \int_{2^{-k+1} R \leq d\left(g, g^{\prime}\right) \leq 2^{-k} R} \frac{\beta}{d\left(g, g^{\prime}\right)^{Q-2}} d H\left(g^{\prime}\right) \\
& \leq \beta \sum_{k=0}^{\infty}\left(\frac{2^{k+1}}{R}\right)^{Q-2} \mid B\left(g, 2^{-k} R \mid \leq C R^{2},\right.
\end{aligned}
$$

where in the last inequality we have used (2.8). This proves the first part of the lemma. To prove the second part we consider now the solution to the problem $\mathcal{L} f=-1$ in $B=B(e, 1), f=0$ on $\partial B$. by the hypoellipticity of $\mathcal{L}$ we have $f \in$ $C^{\infty}(B)$, and therefore $C=|X f(e)|<\infty$. The function $\psi_{R}(g)=R^{-2} \psi\left(\delta_{R}(g)\right)$ is also a solution to the same problem, therefore by Theorem 6.2 we must have $\psi_{R} \equiv f$ in $\bar{B}$. In particular, we have $\left|X \psi_{R}(e)\right|=|X f(e)|=C$. Since $|X \psi(e)|=$ $R\left|X \psi_{R}(e)\right|$, the conclusion follows. 
The following two theorems constitute a somewhat unexpected generalization of corresponding classical results.

Theorem 5.3 (Optimal bound for the horizontal gradient). Consider a normalized solution $w$ in $B$. There exists a constant $C_{1}>0$, depending on $\mathbf{G}$, such that for every $g_{o} \in B$ one has

$$
\left|X w\left(g_{o}\right)\right| \leq C_{1} \sqrt{w\left(g_{o}\right)} .
$$

Proof. Thanks to Theorem 4.3, we have $w \in \Gamma^{1,1}$, hence in particular $X w$ is continuous in $B$. We begin by proving (5.2) when $g_{o} \in \Omega(w)$. Observe first that there exists a constant $C^{*}>0$ such that if $r=w\left(g_{o}\right)$, then

$$
B\left(g_{o}, \sqrt{C^{*} r}\right) \subset \Omega(w) .
$$

To see this let $\rho=d\left(g_{o}, \Lambda(w)\right)$ and $g_{1} \in \Lambda(w)$ be such that $\rho=d\left(g_{o}, g_{1}\right)$. Thanks to Lemma 4.2 one has for some constant $\bar{C}$

$$
\sup _{B\left(g_{1}, 2 \rho\right)} w \leq \bar{C} \rho^{2}
$$

This gives in particular $r=w\left(g_{o}\right) \leq \bar{C} d\left(g_{o}, \Lambda(w)\right)^{2}$. It is then clear that the choice $C^{*}=(4 \bar{C})^{-1}$ implies (5.3). With $R=\sqrt{C^{*} r}$ consider in $B\left(g_{o}, R\right)$ the function $v=w+\psi$, where $\psi$ is the solution to $\mathcal{L} \psi=-1$ in $B\left(g_{o}, R\right)$, with zero boundary values. Clearly, $v$ is $\mathcal{L}$-harmonic and non-negative in $B\left(g_{o}, R\right)$. By Theorems 6.5 and 6.4 and Lemma 5.2 one obtains

$$
\left|X v\left(g_{o}\right)\right| \leq \frac{C}{R} v\left(g_{o}\right) \leq \frac{C}{R}\left[w\left(g_{o}\right)+\psi\left(g_{o}\right)\right] \leq \frac{C}{R}\left[r+C R^{2}\right] \leq C \sqrt{r} .
$$

Finally, using Lemma 5.2 again we conclude

$$
\left|X w\left(g_{o}\right)\right| \leq\left|X v\left(g_{o}\right)\right|+\left|X \psi\left(g_{o}\right)\right| \leq C \sqrt{r} .
$$

This establishes (5.2) when $g_{o} \in \Omega(w)$. By the continuity of $w$ and $X w$, this estimate can now be extended to $\partial \Omega(w)$. On the other hand, (5.2) is trivially true in the interior of the coincidence set, therefore the theorem is fully proved.

Theorem 5.4 (Maximum growth). Let $w$ be a normalized solution to the obstacle problem in $B$. There exists $C_{2}>0$, depending only on $\mathbf{G}$, such that for $g_{o} \in \overline{\Omega(w)}$

$$
\sup _{B\left(g_{o}, r\right)} w \geq C_{2} r^{2}
$$


Before we can prove Theorem 5.4 we need to recall a symmetry result discovered in [12] which will play an important role.

Theorem 5.5. Let $f \in L^{1}(0, \infty)$ be a function with bounded support and such that

$$
M=\int_{0}^{\infty} f(s) \frac{d s}{s}<+\infty .
$$

For a fixed $g_{o} \in \mathbf{G}$ consider the function

$$
v(g)=\int_{\mathbf{G}} f\left(\frac{1}{\Gamma\left(g^{\prime}, g_{o}\right)}\right) \frac{\left|X \Gamma\left(g^{\prime}, g_{o}\right)\right|^{2}}{\Gamma\left(g^{\prime}, g_{o}\right)^{2}} \Gamma\left(g, g^{\prime}\right) d H\left(g^{\prime}\right), \quad g \in \mathbf{G}
$$

which satisfies the equation

$$
\mathcal{L} v=-f\left(\frac{1}{\Gamma\left(\cdot, g_{o}\right)}\right) \frac{\left|X \Gamma\left(\cdot, g_{o}\right)\right|^{2}}{\Gamma\left(\cdot, g_{o}\right)^{2}} .
$$

Under such assumptions $v$ is constant on the level sets of $\Gamma\left(\cdot, g_{o}\right)$, i.e., there exists an absolutely continuous function $v^{*}:(0, \infty) \rightarrow \mathbb{R}$ such that

$$
v(g)=v^{*}\left(\frac{1}{\Gamma\left(\mathcal{g}, g_{o}\right)}\right) .
$$

Moreover, if $f \geq 0$, then $v$ attains its maximum value $M$ in $g_{o}$.

The crucial aspect of Theorem 5.5 for us is that, for a suitable choice of $f$, the function $v$ will serve as a ad hoc replacement of the quadratic polynomials in Caffarelli's original proof for Laplace equation. Moreover, $v$ has symmetry, in the sense that it is constant on the level sets of the fundamental solution of the subLaplacian, and this property will enable us to locate its maximum values. This is not possible, instead, with the apparently more natural choice of the solution $u$ to $\mathcal{L} u=-1$.

Proof of Theorem 5.4. By continuity it is enough to assume $g_{o} \in \Omega(w)$. We will use as a barrier the function $v$ in Theorem 5.5 with the choice $f(s)=$ $s^{2 /(Q-2)} X_{\left(0, r^{Q-2}\right)}(s)$, where $Q$ is the homogeneous dimension of $\mathbf{G}$ and $X_{\left(0, r^{Q-2}\right)}$ denotes the indicator function of the interval $\left(0, r^{Q-2}\right)$. We note explicitly that, since $f \geq 0$, then according to Theorem 5.5 the maximum value of $v$ is attained at the "center" $g_{o}$ of the level set of $\Gamma$

$$
\mathcal{E}\left(g_{o}, r\right)=\left\{g \in \mathbf{G} \mid \Gamma\left(g, g_{o}\right)>r^{2-Q}\right\} .
$$

In view of (5.5), such maximum is given by

$$
v\left(g_{o}\right)=M=\int_{0}^{r^{Q-2}} s^{2 /(Q-2)} \frac{d s}{s}=\frac{Q-2}{2} r^{2} .
$$


By the homogeneity properties of $\Gamma$ recalled in the proof of Lemma 5.2, and the fact that the vector fields $X_{1}, \ldots, X_{m}$ are homogeneous of degree one with respect to the group dilations, we obtain

$$
\left|X \Gamma\left(\mathfrak{g}, g^{\prime}\right)\right| \leq \frac{C}{d\left(g, g^{\prime}\right)^{Q-1}}, \quad g, g^{\prime} \in \mathbf{G}, g \neq g^{\prime},
$$

where $C=C(\mathbf{G})>0$. We note also that, thanks to (5.1) the level sets $\mathcal{E}\left(g_{o}, r\right)$ are trapped between two pseudo-balls of radii proportional to $r$, and therefore the Haar measure of $\mathcal{E}\left(g_{o}, r\right)$ is itself proportional to $r^{Q}$. Combining (5.1) with (5.10), we obtain from (5.7) for every $g \neq g_{o}$

$$
|\mathcal{L} v(g)|=\left|\left(\frac{1}{\Gamma\left(\mathcal{g}, g_{o}\right)}\right)^{2 /(Q-2)} \frac{\left|X \Gamma\left(g, g_{o}\right)\right|^{2}}{\Gamma\left(g, g_{o}\right)^{2}}\right| \leq K,
$$

for some $K=K(\mathbf{G})>0$. We consider next the function $\zeta=K w+v-v\left(g_{o}\right)$, which satisfies $\mathcal{L} \zeta \geq 0$ in $A=\Omega(w) \cap \mathcal{E}\left(g_{o}, r\right)$. Since $\zeta\left(g_{o}\right)>0$, by Bony's maximum principle $\zeta$ must attain a strictly positive maximum on $\partial A$. However, we have $\zeta \leq 0$ on $\partial \Omega(w) \cap\left\{g \in \mathbf{G} \mid \Gamma\left(\boldsymbol{g}, g_{o}\right)>r^{2-Q}\right\}$, hence the maximum occurs at some point $g_{1} \in \Omega(w) \cap \partial\left\{g \in \mathbf{G} \mid \Gamma\left(g, g_{o}\right)>r^{2-Q}\right\}$. One concludes

$$
0<\zeta\left(g_{1}\right)=K w\left(g_{1}\right)+v\left(g_{1}\right)-v\left(g_{o}\right) .
$$

From the proof of Theorem 5.5 in [12] we deduce that in the present case the symmetric part of $v$ is given by

$$
v^{*}(t)=\frac{1}{t} \int_{0}^{t} s^{2 /(Q-2)} d s=\frac{Q-2}{Q} t^{2 /(Q-2)} .
$$

By the remarkable property (5.8) we now infer the crucial information

$$
v\left(g_{1}\right)=v^{*}\left(\frac{1}{\Gamma\left(g_{1}, g_{o}\right)}\right)=v^{*}\left(r^{Q-2}\right)=\frac{Q-2}{Q} r^{2} .
$$

The latter equation and (5.9) allow to conclude

$$
v\left(g_{1}\right)-v\left(g_{o}\right)=-\frac{(Q-2)^{2}}{2 Q} r^{2} .
$$

Substituting this information in (5.11) we conclude

$$
w\left(g_{1}\right) \geq \frac{(Q-2)^{2}}{2 Q K} r^{2} .
$$

This completes the proof of the theorem. 
In the statement of the next theorem we adopt the notation $|E|$ to indicate the Haar measure $H(E)$ of a measurable set $E \subset \mathbf{G}$.

Theorem 5.6 (Uniform positive density of $\Omega(w)$ along the free boundary). There exists a universal constant $A>0$ such that for every $g_{o} \in \partial \Omega(w)$ and any $r>0$

$$
\left|B\left(g_{o}, r\right) \cap \Omega(w)\right| \geq A\left|B\left(g_{o}, r\right)\right| .
$$

In particular, the lower density

$$
\underline{D}\left(\Omega(w), g_{o}\right)=\liminf _{r \rightarrow 0} \frac{\left|B\left(g_{o}, r\right) \cap \Omega(w)\right|}{\left|B\left(g_{o}, r\right)\right|}
$$

of $\Omega(w)$ at $g_{o}$ satisfies $\underline{D}\left(\Omega(w), g_{o}\right) \geq A$.

Proof. Consider $g_{1} \in B\left(g_{o}, r / 2\right) \cap \Omega(w)$ such that $w\left(g_{1}\right)=\sup _{B\left(g_{o}, r / 2\right)} w$. By Theorem 5.4 one has $w\left(g_{1}\right) \geq C^{*} r^{2}$. On the other hand Theorem 5.3 and Lemma 4.2 give $\sup _{B\left(g_{o}, r\right)}|X w| \leq C^{* *} r$. By Theorem 4.1 we infer for any $g \in B\left(g_{1}, \delta r\right)$

$$
w\left(g_{1}\right)-w(g) \leq \delta \bar{C} r^{2} .
$$

These estimates guarantee that

$$
w(g) \geq\left[C^{*}-\delta \bar{C}\right] r^{2} \geq \frac{1}{2} r^{2}
$$

provided that $\delta$ is sufficiently small. This implies that $B\left(g_{1}, \delta r\right) \subset B\left(g_{o}, r\right) \cap$ $\Omega(w)$ and therefore

$$
\left|B\left(g_{o}, r\right) \cap \Omega(w)\right| \geq\left|B\left(g_{1}, \delta r\right)\right|=C(\mathbf{G}, \delta) r^{Q}=A\left|B\left(g_{o}, r\right)\right| .
$$

Corollary 5.7. The free boundary has zero Haar measure, i.e.,

$$
|\partial \Omega(w)|=0 .
$$

Proof. Consider the indicator function $\chi_{\Omega}$ of the positivity set $\Omega=\Omega(w)$. Since $\chi_{\Omega} \in L_{\text {loc }}^{1}(\mathbf{G})$, by Lebesgue differentiation theorem for spaces of homogeneous type (see [40]) we know that $d H$-a.e. point of $\mathbf{G}$ is a Lebesgue point. Therefore, the set

$$
S=\left\{g \in \mathbf{G} \mid \frac{|B(g, r) \cap \Omega|}{|B(g, r)|}=\frac{1}{|B(g, r)|} \int_{B(g, r)} \chi_{\Omega}+\chi_{\Omega} \quad \text { as } \quad r \rightarrow 0\right\}
$$

has zero $d H$ measure. On the other hand, Theorem 5.6 proves that $\partial \Omega \subset S$, therefore the conclusion follows. 


\section{APPENDIX: SOME KNOWN RESUlTS FROM \\ Partial Differential EQUations and Potential Theory}

In this appendix we collect various known results that are needed in the proofs of the main theorems in Sections 3 and 5.

Theorem 6.1 (Bony's strong maximum principle [2]). Let $\Omega$ be a connected open set in a Carnot group G. Assume that $c \leq 0$ in $\Omega$ and that $c \in C(\bar{\Omega})$. If $u \in C^{2}(\Omega)$ satisfies

$$
\mathcal{L} u+Y u+c u \leq 0 \quad \text { in } \Omega,
$$

then $u$ cannot achieve a non-positive infimum at an interior point, unless $u \equiv$ const in $\Omega$. Here, $Y$ denotes a smooth vector field on $\mathbf{G}$.

The following result is an important consequence of the work [2].

Theorem 6.2. Let $D \subset \mathbf{G}$ be a connected, bounded open set, and $\varphi \in C(\partial D)$. There exists a unique $\mathcal{L}$-harmonic function $H_{p}^{D}$ which solves $(D P)$ in the sense of Perron-Wiener-Brelot. Moreover, $H_{\varphi}^{D}$ satisfies

$$
\sup _{D}\left|H_{\varphi}^{D}\right| \leq \sup _{\partial D}|\varphi| .
$$

Theorem 6.3 (Weak Harnack inequality [7]). Consider an open set D $\subset \mathbf{G}$ and let $u \in \mathcal{L}^{1,2}(D)$ be a non-negative $\mathcal{L}$-superharmonic function in $D$. There exists $q_{o}=q_{o}(\mathbf{G})>0$ such that for every $B\left(g_{o}, R\right) \subset \bar{B}\left(g_{o}, 2 R\right) \subset D$ and $0<q<q_{o}$ one has

$$
\left(\frac{1}{\left|B\left(g_{o}, R\right)\right|} \int_{B\left(g_{o}, R\right)} u^{q} d H\right)^{1 / q} \leq C \operatorname{essinf}_{B\left(g_{o}, s R\right)} u,
$$

for every $0<s<1$. Here, $C=C(\mathbf{G}, s, q)$.

Theorem 6.4 (Harnack inequality [2], see also [12] and [7]). There exists a positive constant $C=C(\mathbf{G})$ such that if $u$ is a non-negative $\mathcal{L}$-harmonic function in $B\left(g_{o}, 4 R\right) \subset \mathbf{G}$, then

$$
\sup _{B\left(g_{o}, R\right)} u \leq C \inf _{B\left(g_{o}, R\right)} u .
$$

Theorem 6.5 (Schauder type interior estimates, see [43] or also [14]). Let $D \subset \mathbf{G}$ be an open set and suppose that $w$ is $\mathcal{L}$-harmonic in $D$. For every $g \in D$ and $r>0$ for which $\bar{B}(g, r) \subset D$, one has for $s \in \mathbb{N}$

$$
\left|X_{j_{1}} X_{j_{2}} \cdots X_{j_{s}} w(g)\right| \leq \frac{C}{r^{s}} \frac{\max }{\bar{B}(g, r)}|w|,
$$

for $j_{i} \in\{1, \ldots, m\}, i=1, \ldots, s$, and for some constant $C=C(\mathbf{G}, s)>0$. 


\section{REFERENCES}

[1] H. BREZIS \& D. KINDERLEHRER, The smoothness of solutions to nonlinear variational inequalities, Indiana U. Math. J. 23 (9) (1974), 831-844.

[2] J. M. BONY, Principe du maximum, inégalité de Harnack et unicité du problème de Cauchy pour les opérateurs elliptiques dégénérés, Ann. Inst. Fourier, Grenoble, 19.1 (1969), 277-304.

[3] R. W. BROCKETT, Control theory and singular Riemannian geometry, New directions in applied mathematics (Cleveland, Ohio, 1980), p. 11-27, Springer, New York-Berlin, 1982.

[4] L. A. Caffarelli, The obstacle problem revisited, J. Fourier Anal. Appl. 4 (4\& 5) (1998), 383-402.

[5] L. A. Caffarelli \& D. Kinderlehrer, Potential methods in variational inequalitites, J. Anal. Math. 37 (1980), 285-295.

[6] A. P. CALDERÓN, On the differentiability of absolutely continuous functions, Rivista Mat. Univ. Parma 2 (1951), 203-213.

[7] L. Capogna, D. Danielli \& N. Garofalo, An embedding theorem and the Harnack inequality for nonlinear subelliptic equations, Comm. Part. Diff. Eq. 18 (9 \& 10) (1993), 17651794.

[8] L. Capogna, D. Danielli \& N. Garofalo, Subelliptic mollifiers and a basic pointwise estimate of Poincaré type, Math. Zeit. 226 (1997), 147-154.

[9] C. CARATHÉOdory, Untersuchungen über die Grundlangen der Thermodynamik, Math. Ann. 67 (1909), 355-386.

[10] L. CESARI, Sulle funzioni assolutamente continue in due variabili, Ann. Scuola Norm. Super. Pisa (2) 10 (1941), 91-101.

[11] G. S. CHIRIKJIAN \& A. B. KYATKIN, Engineering Applications of Noncommutative Harmonic Analysis, CRC Press, Boca Raton, London, New York, Washington, D.C., 2001.

[12] G. CitTI, N. GAROFALO \& E. LANCONELLI, Harnack's inequality for sum of squares of vector fields plus a potential, Amer. J. of Math. 3115 (1993), 699-734.

[13] W. L. CHOW, Über Systeme von linearen partiellen Differentialgleichungen erster Ordnung, Math. Annalen 117 (1939), 98-105.

[14] D. Danielli \& N. Garofalo, Geometric properties of solutions to subelliptic equations in nilpotent Lie groups, Lect. Notes in Pure and Appl. Math., "Reaction Diffusion Systems", Trieste, October 1995, Ed. G. Caristi Invernizzi, E. Mitidieri, Marcel Dekker 194 (1998).

[15] G. C. Evans, On potentials of positive mass I, Trans. Amer. Math. Soc. 37 (1935), 226-253.

[16] L. C. Evans \& R. F. GARIEPy, Measure Theorey and Fine Properties of Functions, Studies in Adv. Math., CRC Press, Boca Raton, Ann Arbor, London, 1992.

[17] H. Federer, Geometric Measure Theory, Classics in Mathematics, reprint of the 1969 edition, Springer, 1996.

[18] G. Folland, Subelliptic estimates and function spaces on nilpotent Lie groups, Ark. Math. 13 (1975), 161-207.

[19] G. B. Folland \& E. M. STEIN, Estimates for the $\bar{\partial}_{b}$ Complex and Analysis on the Heisenberg Group, Comm. Pure Appl. Math. 27 (1974), 429-522.

[20] G. B. Folland \& E. M. Stein, Hardy Spaces on Homogeneous Groups, Princeton Univ. Press., (1982).

[21] B. Franchi, G. Lu \& R. L. Wheeden, Representation formulas and weighted Poincaré inequalities for Hörmander vector fields, Ann. Inst. Fourier 45 (1995), no. 2, 577-604. 
[22] B. Franchi, R. Serapioni \& F. Serra Cassano, Approximation and imbedding theorems for weighted Sobolev spaces associated with Lipschitz continuous vector fields, Boll. Un. Mat. Ital. B (7) 11 (1997), no. 1, 83-117.

[23] J. FREHSE, On the regularity of solutions of a second order variational inequality, Boll. U.M.I. (4) 6 (1972), 312-315.

[24] N. Garofalo, Analysis and Geometry of Carnot-Caratheodory Spaces, With Applications to Pde's, Birkhäuser, book in preparation.

[25] N. Garofalo \& D. M. NHIEU, Lipschitz continuity, global smooth approximations and extension theorems for Sobolev functions in Carnot-Carathéodory spaces, J. Analyse Math. 74 (1998), 67-97.

[26] J. Heinonen, T. KilpeläInen \& O. Martio, Nonlinear Potential Theory of Degenerate Elliptic Equations, Oxford Math. Monographs, Clarendon Press, 1993.

[27] R. M. Hervé \& M. Hervé, Les functions surharmoniques dans l'axiomatique de M. Brelot associées à un operateur elliptique dégénéré, Ann. Inst. Fourier 22 (2) (1972), 131-145.

[28] H. HÖRMANDER, Hypoelliptic second-order differential equations, Acta Math. 119 (1967), 147171.

[29] D. Kinderlehrer \& G. STAmpacChia, An introduction to variational inequalities and their applications, Academic Press, 1980.

[30] A. Kolmogorov, Zufällige Bewegungen, Ann. Math. 35 (1934), 116-117.

[31] H. LEWY, An example of a smooth linear partial differential equation without solution, Ann. of Math., (2) 66 (1957), 155-158.

[32] J. L. Lions \& G. Stampacchia, Variational inequalities, Comm. Pure Appl. Math. 20 (1967), 493-519.

[33] G. LU, Weighted Poincaré and Sobolev inequalities for vector fields satisfying Hörmander's condition and applications, Rev. Mat. Iberoamericana 8 (1992), no. 3, 367-439.

[34] R. Monti \& F. SERra CaSSANO, Surface measures in Carnot-Carathéodory spaces, Calculus of Variations and PDE, to appear.

[35] P. PANSU, Métriques de Carnot-Carathéodory et quasi-isométries des espaces symétriques de rang un, Ann. of Math. (2) 129 (1989), 1, 1-60.

[36] L. P. ROTHSCHILD \& E. M. STEIN, Hypoelliptic differential operators and nilpotent groups, Acta Math. 137 (1976), 247-320.

[37] G. STAMPACCHIA, Formes bilinéaires coercitives sur les ensembles convexes, C.R. Acad. Sci. Paris 258 (1964), 4413-4416.

[38] E. M. STEIn, Singular Integrals and Differentiability Properties of Functions, Princeton Univ. Press, 1970.

[39] E. M. STEIN, Some problems in harmonic analysis suggested by symmetris spaces and semisimple groups, Proc. Int. Congress Math. Nice, 1970, Gauthier-Villars, Paris, 1971, 173-179.

[40] E. M. STEIN, Harmonic Analysis: Real Variable Methods, Orthogonality and Oscillatory Integrals, Princeton Univ. Press, 1993.

[41] H. J. SussmanN, Optimal control theory and piecewise analyticity of the distance function for some real-analytic sub-Riemannian metrics, Optimization and nonlinear analysis (Haifa, 1990), Pitman Res. Notes Math. Ser., Longman Sci. Tech., Harlow 244 1992, 298-310.

[42] V. S. VARAdarajan, Lie Groups, Lie Algebras, and Their Representations, Springer-Verlag, New York, Berlin, Heidelberg, Tokyo, 1974. 
[43] N. Th. Varopoulos, L. Saloff-Coste \& T. Coulhon, Analysis and Geometry on Groups, Cambridge U. Press, 1992.

[44] F. VASILESCO, Sur la continuité du potentiel à travers des masses et la démonstration d'une lemme de Kellogg, C. R. Acad. Sci. Paris 200 (1935), 1173-1174.

[45] S. VODOP'yANOV, $\mathcal{P}$-differentiability on Carnot groups in different topologies and related topics, Proceedings on Analysis and Geometry (Novosibirsk Akademgorodok, 1999) (Russian), 603670, Izdat. Ross. Akad. Nauk Sib. Otd. Inst. Mat., Novosibirsk, 2000.

DONATELLA DANIELLI:

Department of Mathematics

Purdue University

West Lafayette, IN 47907-1395, U.S.A.

E-MAIL: danielli@math.purdue.edu

Nicola Garofalo:

Department of Mathematics

Purdue University

West Lafayette, IN 47907-1395, U.S.A.

E-MAIL: garofalo@math.jhu.edu

SANDRO SALSA:

Dipartimento di Matematica

Politecnico di Milano

Milano, Italy

E-MAIL: sansal@mate.polimi.it

ACKNOWLEDGMENT: The second author was supported in part by NSF Grant No. DMS-9706892, and by NSF Grant No. DMS-0070492

KeY WORDS And PHRASES: Obstacle problem, Free boundaries, Sub-elliptic equations, Carnot groups, Theorems of Rademacher-Stepanov type.

2000 Mathematics Subject Classification: 35J85, 35H20, 43A85

Received: June 23rd, 2000; revised: November 1st, 2001. 